\title{
Norois
}

Environnement, aménagement, société

\section{La Garonne à Toulouse}

Du centre ancien aux communes périurbaines : observation des paysages, évolution et intentionnalités

The Garonne in Toulouse. The old center with suburban towns: observation of landscapes, evolution, and intentionality

\section{Philippe Valette}

\section{(2) OpenEdition}

\section{Journals}

Édition électronique

URL : https://journals.openedition.org/norois/5798

DOI : $10.4000 /$ norois. 5798

ISBN : 978-2-7535-5086-5

ISSN : $1760-8546$

Éditeur

Presses universitaires de Rennes

\section{Édition imprimée}

Date de publication : 31 décembre 2015

Pagination : 81-100

ISBN : 978-2-7535-5084-1

ISSN : 0029-182X

\section{Référence électronique}

Philippe Valette, «La Garonne à Toulouse », Norois [En ligne], 237 | 2015, mis en ligne le 31 décembre 2017, consulté le 28 janvier 2022. URL : http://journals.openedition.org/norois/5798 ; DOI : https:// doi.org/10.4000/norois.5798 


Presses
Universitaires
de Rennes
www.pur-editions.fr

\title{
La Garonne à Toulouse Du centre ancien aux communes périurbaines : observation des paysages, évolution et intentionnalités
}

\author{
The Garonne in Toulouse \\ From the Old Center to Suburban Towns: Observation of Landscapes, Evolution \\ and Intentionality
}

\author{
Philippe Valette
}

Maître de conférences, Université de Toulouse Jean Jaurès, GEODE UMR 5602 CNRS.

(Philippe.valette@univ-tlse2.fr)

\begin{abstract}
Résumé : Les paysages garonnais toulousains, du centre ancien jusqu'aux communes périurbaines sud et nord, sont caractérisés par une diversité de paysages fluviaux liée à l'histoire des relations entre la Garonne et la ville de Toulouse. Dans le centre ancien les quais et digues (créés au XVIII ${ }^{\mathrm{e}}$ siècle et XIX ${ }^{\mathrm{e}}$ siècle) sont constitués de briques roses et font partie de l'identité de la ville. En amont et aval immédiats du front historique, des murs gris en béton composent un tout autre paysage (post 1950). Plus loin encore, dans les communes périurbaines, la valorisation des paysages fluviaux s’exprime par des éléments ponctuels (espaces verts, aires de loisirs...). Cette diversité témoigne d'un traitement paysager différent selon les époques. Dans ce travail, il est question de caractériser les intentionnalités paysagères au cours du temps afin de mieux comprendre l'état actuel des paysages fluviaux.

Plusieurs types d'intentionnalités peuvent être définis et parmi elles, nous nous intéressons plus particulièrement à l'embellissement et la valorisation des paysages fluviaux urbains et périurbains. Pendant de nombreuses années, l'intentionnalité liée à l'embellissement des paysages fluviaux urbains toulousains s'est concentrée dans le centre ancien. Depuis quelques années, ce processus semble s'étendre en amont et en aval du centre-ville. Actuellement, de nombreuses réflexions sont en cours autour du projet « Grand parc Garonne » mené par Toulouse Métropole (CUGT, 2012). Ce projet vise à valoriser les paysages fluviaux et le patrimoine fluvial du centre ancien jusque dans ces communes périurbaines. Dans ces territoires est-il possible de parler d'une intentionnalité paysagère comparable à celle que nous observons en centre-ville? Existe-t-il une continuité dans la valorisation des paysages fluviaux toulousains?
\end{abstract}

\begin{abstract}
Landscapes Garonne Toulouse, the old center south and north to peri-urban municipalities are characterized by diverse river landscapes linked to the history of relations between the Garonne and the city of Toulouse. In the old center docks and breakwaters (created in the eighteenth and nineteenth centuries) are made of red brick and are part of the identity of the city. Upstream and immediate downstream of the historical front, gray concrete walls make up a completely different landscape (post 1950). Further still, in suburban towns, development of river landscapes is expressed by one-off items (green spaces, recreational areas...). In this work, it comes to characterize the landscape intentionalities over time to better understand the current state of river landscapes.

Several types of intentionality can be defined and among them, we are particularly interested in the beautification and enhancement of urban and suburban river landscapes. For many years, intentionality related to the beautification of the Toulouse urban river landscapes concentrated in the old center. In recent years, this process seems to extend upstream and downstream of the city center. Currently, many reflections are underway around the project "Garonne Grand Park" led by Toulouse Métropole (CUGT, 2012). This project seeks to develop the river landscapes and River Heritage old center into the suburban municipalities. In these areas it is possible to speak of a landscape intentionality comparable to that which we observe in the city center? Is there continuity in the valuation of Toulouse river landscapes?
\end{abstract}


Mots clés : Garonne - paysage fluviaux urbains - Toulouse - périurbain - intentionnalité paysagère - dynamiques paysagères

Keywords: Garonneriver - urbanlandscape - Toulouse - suburban - landscape intentionality - landscape dynamics

\section{INTRODUCTION}

De nos jours, après des décennies d'abandon, les intentions des acteurs urbains visent la reconquête des paysages fluviaux en ville. Ce vaste mouvement s'effectue un peu partout en France et dans le monde. On ne compte plus les exemples de retour au fleuve à la fois dans les pays occidentaux et les pays en voie de développement. En Europe, il est possible de citer : la Seine à Paris, le Rhône à Lyon, la Garonne à Bordeaux, l'Isar à Munich... Ailleurs dans le monde, les exemples sont nombreux comme sur le Saint-Laurent à Québec, l'Hudson à New York, le Sabarmati à Ahmedabad en Inde, la rivière Hai à Tianjin en Chine, le Paraguay à Asunción, le Rio de la Plata à Buenos Aires... Le moindre petit cours d'eau en ville fait l'objet d'une intentionnalité paysagère esthétique à partir de projets réalisés par des cabinets de paysagistes (Topos, 2009; Paisea, 2009). À partir des années 1990, la plupart des réflexions se cristallise autour des fronts urbains anciens qui font l'objet de projets d'aménagements autour d'une valorisation des berges. Mais, depuis une dizaine d'années, l'échelle d'analyse change et s'élargit considérablement à l'image du projet « Paris, Métropole sur Seine » (APUR, 2010). Il s'agit d'englober le retour aux cours d'eau dans un territoire plus vaste que le seul centre ancien. Ce renouveau tend alors à s'exprimer sur plusieurs kilomètres en amont et en aval des villes anciennes. En France, plusieurs travaux traitent de ces questions (Chaline, 1994 ; Salles, 2003; DGUHC, 2006; Bonin, 2007; Beucher, 2009; Romain, 2010; Dournel et al., 2011 ; Dournel, 2010 ; Carré, 2011 ; Carré, Deutsch, 2015). Le colloque intitulé « Rivières et métropoles européennes » (juillet 2016), organisé par l'IREST (Institut de Recherche et d'Etudes Supéruieures du Tourisme) à la Sorbonne, insiste d'ailleurs sur ce changement d'échelle dans la fréquentation ludique des rivières urbaines.

Qu'en est-il de de ce vaste mouvement au niveau de la ville de Toulouse? Le retour au fleuve à Toulouse doit être replacé dans son contexte géohistorique afin de connaître la construction pro- gressive des paysages fluviaux urbains au cours de l'histoire (de la fin du Moyen Âge à aujourd'hui). Les bords de la Garonne à Toulouse ont-ils fait l'objet de valorisations et quelles sont les intentionnalités paysagères au cours du temps? Les réflexions actuelles relatives au projet « Grand parc Garonne » menées par Toulouse Métropole élargissent considérablement le territoire d'intervention à l'image des autres exemples français (Communauté Urbaine du Grand Toulouse CUGT, 2012). Face à ce changement d'échelle, il est nécessaire dans une première partie de définir notre approche et de caractériser les paysages fluviaux de ce vaste territoire. Dans une seconde partie, nous détaillerons leur construction progressive en questionnant la part d'intentionnalité paysagère à partir de la fin du Moyen Âge. Enfin, dans la dernière partie, il s'agira d'évoquer la diffusion du renouveau actuel de la Garonne du centre ancien vers les espaces périphériques amont et aval.

\section{INTENTIONNALITÉS DES PAYSAGES FLUVIAUX URBAINS TOULOUSAINS : DU CENTRE ANCIEN À SA PÉRIPHÉRIE}

Notre travail s'articule autour de l'utilisation de la notion d'intentionnalité paysagère appliquée au terrain de la Garonne à Toulouse dans un contexte urbain et périurbain. Travailler sur cette question selon une démarche spatio-temporelle impose de revenir sur des éléments de définition de cette notion.

\section{Une clef de lecture : la notion d'intentionnalité paysagère}

L'intentionnalité en philosophie correspond au caractère propre qu'a la conscience d'être toujours tendue vers un but, un objet (définition du Larousse). Plusieurs auteurs ont travaillé sur cette notion à travers de multiples approches (Barraud, 2007 : Debarbieux, 2007; Parent, 2005; Candau, 2003). Parent (2005) utilise cette notion pour l'interprétation des œuvres picturales. Ainsi, parler de l'intention d'un tableau, «c'est décrire les conditions 
d'apparition de ce tableau, en se fondant sur l'hypothèse que celui qui l'a fait agissait de manière intentionnelle » (Baxandall, 1991). Il s'agit donc d'examiner la façon dont la décision a été prise de faire un objet et d'étudier les conditions dans lesquelles l'entreprise a été menée (Parent, 2005). Rendre compte d'une intention paysagère, c'est donc analyser les moyens à disposition et les objectifs poursuivis pour aménager, valoriser un paysage.

Dans le cadre des paysages fluviaux urbains et périurbains toulousains, il s'agit de comprendre les intentionnalités paysagères multiples, à la fois dans l'espace et dans le temps. Relier intentionnalités paysagères et temporalités permet d'étudier les problématiques d'aménagement au cours du temps. Barraud (2007) propose une grille d'interprétation des intentions des aménagements des paysages fluviaux depuis la période moderne dans les vallées de l'ouest de la France. Dans ce cadre-là, il propose le terme de « régime d'intentionnalité » qui correspond aux intentions d'aménagement des paysages. L'aménagement, la valorisation ou l'amélioration d'un paysage sont bien intentionnels et sont orientés vers un type d'usage. Coupler temporalités et intentionnalités paysagères permet de reconstituer la dynamique des paysages fluviaux et de connaître la multiplicité des intentionnalités au cours du temps. Il s'agit donc à travers l'intentionnalité de reconstituer les trajectoires paysagères (Barraud, 2007).

Par conséquent, pour évoquer la part d'intentionnalité paysagère au sein des paysages fluviaux urbains toulousains, nous adoptons une démarche spatio-temporelle et diachronique. Notre démarche géohistorique s'étend de la fin du Moyen Âge à aujourd'hui, à travers l'analyse de documents anciens (images, textes, cartes et plans). Dans ce cadre, nous accordons une place particulière à la photographie ancienne et la rephotographie systématique à partir de sites identifiés sur les bords de la Garonne à Toulouse. La mise en place d'une démarche d'observatoire des paysages de la Garonne à travers l'utilisation de séries photographiques nous permet de montrer les permanences et les mutations des paysages fluviaux urbains toulousains depuis la fin du XIX ${ }^{\mathrm{e}}$ siècle [http://opgaronne.univ-tlse2.fr/]. Cette approche, en cours de développement, permettra à partir de rephotographies actuelles de saisir les évolutions futures du retour au fleuve à Toulouse et sa périphérie.
Les paysages fluviaux urbains liés au centre ancien de la ville de Toulouse ont fait l'objet de plusieurs travaux (Valette, Carozza, 2013; Valette et al, 2013; Papillault et al., 2012; Valette, Carozza, 2011). Peu d'études traitent des espaces périphériques périurbains situés au nord et au sud de la ville, de sorte qu'il est nécessaire de définir et caractériser l'ensemble de ces territoires

\section{La Garonne au centre du territoire toulousain}

Les réflexions actuelles liées au «Grand parc Garonne » s'intéressent à un territoire plus vaste que le centre ancien toulousain (CUGT, 2012). Il semble aujourd'hui s'imposer dans le projet de retour au fleuve de l'agglomération toulousaine. Mais quel est ce territoire? Celui de Toulouse Métropole? Celui de l'aire urbaine toulousaine? Cette question a fait l'objet de plusieurs réflexions (Papillault et al., 2012; CUGT, 2012). Le territoire qui s'étend de Portet-sur-Garonne au Sud jusqu'aux environs de Grenade-sur-Garonne au Nord semble s'imposer (figure 1). «Il s'est détaché l'idée intuitive que le territoire Garonne pertinent de la métropole fonctionnait dans le sens longitudinal de la confluence de l'Ariège à Portet-sur-Garonne jusqu'à la confluence de l'Hers-Save à Grenade-sur-Garonne soit un parcours de méandres de 41700 mètres » (Papillault et al., 2012). Toulouse Métropole ${ }^{1}$, quant à elle, réduit quelque peu ce territoire de Toulouse à SaintJory, « sur près de $32 \mathrm{~km}$, ce territoire constitue le socle de paysages remarquables » (CUGT, 2012).

Jusqu'à la fin du XvIII siècle, il était relativement facile de délimiter les paysages fluviaux urbains puisque les villes étaient pour la plupart encloses de remparts. Les portes de la ville, situées dans les remparts, étaient donc des portes qui séparaient le monde urbain du monde rural. Aujourd'hui, à cause de l'urbanisation et de la périurbanisation, les limites sont beaucoup plus distendues. Plus on s'éloigne de la ville, plus les paysages ruraux dominent, tout en restant sous influence urbaine. « La fin de la porte

\footnotetext{
1. Le District du Grand Toulouse est créé en 1992 et se composait de 13 communes. Cette structure devient en 2001 la Communauté d'Agglomération du Grand Toulouse, composée de 21 communes. En 2012, elle devient la Communauté Urbaine de Toulouse Métropole avec 33 communes. En 2015, Toulouse Métropole la remplace. Il s'agit d'un établissement Public de Coopération Intercommunale (EPCI) et se compose de 37 communes.
} 
de la ville, corollaire du mur, a fait perdre, non sans nostalgie, la précision des contours, la netteté du paysage urbain dès la fin du XVII ${ }^{\text {e }}$ siècle, préparant l'agglomération. L'entrée dans la grande ville devient insensible, on ne peut plus la souligner, ni même la traiter de façon monumentale, la croissance des villes rend vaine toute tentative de poser des bornes » (Lamarre, 2006).

Face à ces deux réflexions sur la bonne échelle d'intervention dans le retour au fleuve, notre terrain d'étude concerne donc le paysage fluvial sous influence toulousaine. Il s'agit des territoires composant l'EPCI Toulouse Métropole (Établissement Public de Coopération Intercommunale), auxquels il faut ajouter 3 communes en amont (Portet-surGaronne, Vieille Toulouse, Pinsaguel) et une commune en aval (Grenade-sur-Garonne). Ce vaste territoire compose l'aire urbaine toulousaine. Touché par la périurbanisation, il est susceptible d'incorporer et de retranscrire une intentionnalité paysagère dans l'aménagement des berges de la Garonne (figure 1). De l'amont vers l'aval, il est composé des communes de Pinsaguel, Portet-sur-Garonne, Vieille Toulouse, Toulouse, Blagnac, Beauzelle, Fenouillet,
Gagnac-sur-Garonne, Seilh, Saint-Jory, Merville et Grenade-sur-Garonne.

\section{Un territoire sous l'emprise de Toulouse}

Ce vaste territoire a été dénommé par certains auteurs comme "parenthèse toulousaine » (Valley, 2011) car, à son arrivée à Toulouse, la Garonne décrit une large boucle. La ville est située au point le plus à l'est sur le tracé du fleuve. Cette "parenthèse », à partir de la confluence de l'Ariège, appartient au tronçon de la moyenne Garonne toulousaine (compris entre la confluence de l'Ariège et la confluence du Tarn). En amont de Toulouse, le fleuve perd une partie de son caractère montagnard au profit d'un élargissement de la vallée où son débit atteint 202 m³/s (SMEPAG, 1989; Valette et al, 2014). La pente de la vallée favorise les effets des crues et des inondations, qui lui ont valu l'appellation par certains auteurs de "Garonne débordante ». Le cours de la Garonne a été particulièrement instable au cours de l'histoire et les paysages gardent encore la trace de nombreuses îles, îlots dont certains sont rattachés à la terre ferme aujourd'hui. Cette
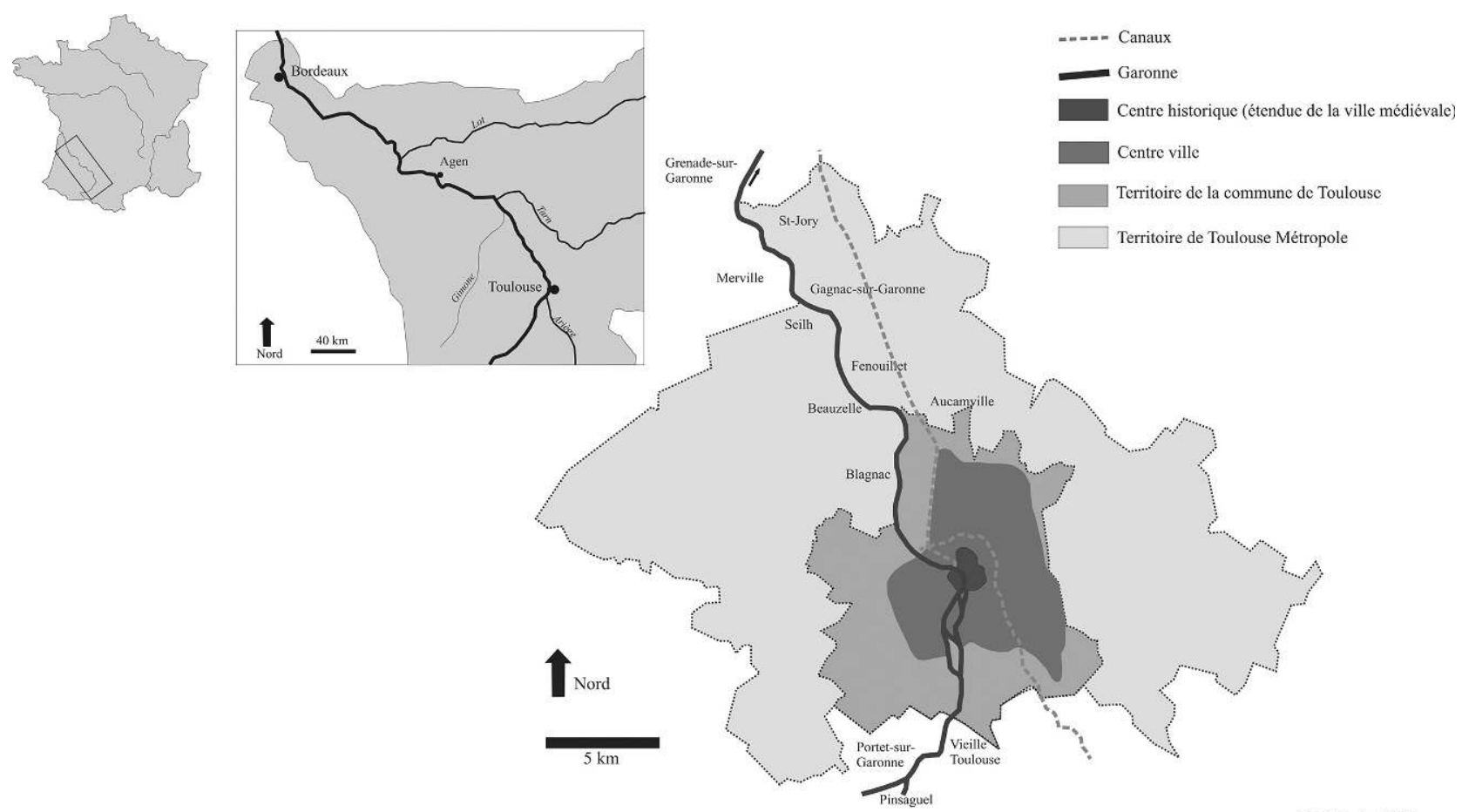

$\mathrm{Ph}$ Valette, 2015

Figure 1 : Situation géographique du terrain d'étude (de Pinsaguel à Grenade-sur-Garonne) Location of the field of study (Pinsaguel to Grenade-sur-Garonne) 
instabilité historique a créé de nombreuses zones humides (bras morts) pour lesquelles la biodiversité est remarquable. Du sud vers le nord, 32 sites ont été identifiés comme patrimoine naturel du « Grand parc Garonne »(ZNIEFF, ZICO, Natura 2000), dont 6 font l'objet d'Arrêtés préfectoraux de protection de biotope (CUGT, 2012). Tous ces dispositifs de protection de la biodiversité se concentrent surtout dans les zones périurbaines, à l'amont et à l'aval de la commune de Toulouse.

D'autre part, l'urbanisation a largement dépassé le simple cadre de la commune de Toulouse. Partout, sur les terrasses alluviales de la rive droite et de la rive gauche, l'urbanisation et la périurbanisation se sont généralisées à travers un habitat pavillonnaire, la mise en place de réseaux de communication (figure 2), de zones artisanales, industrielles et commerciales. La zone inondable reste moins touchée même si autour des bourgs de larges auréoles urbaines se sont développées autour de Fenouillet ou de Gagnac-sur-Garonne par exemple. Ici, la possibilité d'une inondation a limité les implantations d'habitats. Pour combien de temps encore? Ailleurs à Toulouse et Blagnac, l'édification de digues a favorisé l'urbanisation à l'arrière des ouvrages.

\section{Une diversité de séquences paysagères}

De l'amont vers l'aval, il est possible de distinguer 3 séquences paysagères.

La séquence située en amont de la ville dense, caractérisée par une forte dissymétrie (rive droite peu étendue et abrupte) s'étend de la confluence de l'Ariège jusqu'au périphérique situé au pied des coteaux de Pech David (figure 3). La rive droite est constituée des coteaux molassiques de Pech David (260 m d'altitude), alors qu'en face s'étend la plaine inondable et la succession des terrasses alluviales de la Garonne. Cette rive gauche est occupée par une répétition de zones industrielles, commerciales et pavillonnaires. C'est également dans ces territoires de rive gauche que se trouvent les différents axes de communication (voie ferrée, route nationale, autoroute). À proximité du fleuve demeurent encore quelques parcelles de production agricole. La Garonne y est constituée d'îles ou anciennes îles (îlot des lapins, île d'Empalot...). Sur les hauteurs des coteaux, l'habitat pavillonnaire s'est largement développé, chacun recherchant une vue agréable sur le paysage de la vallée de la Garonne.

La deuxième séquence correspond au paysage urbain dense et s'étend des environs d'Empalot jusqu'à Sesquières (figure 3). Ici, l'urbanisation

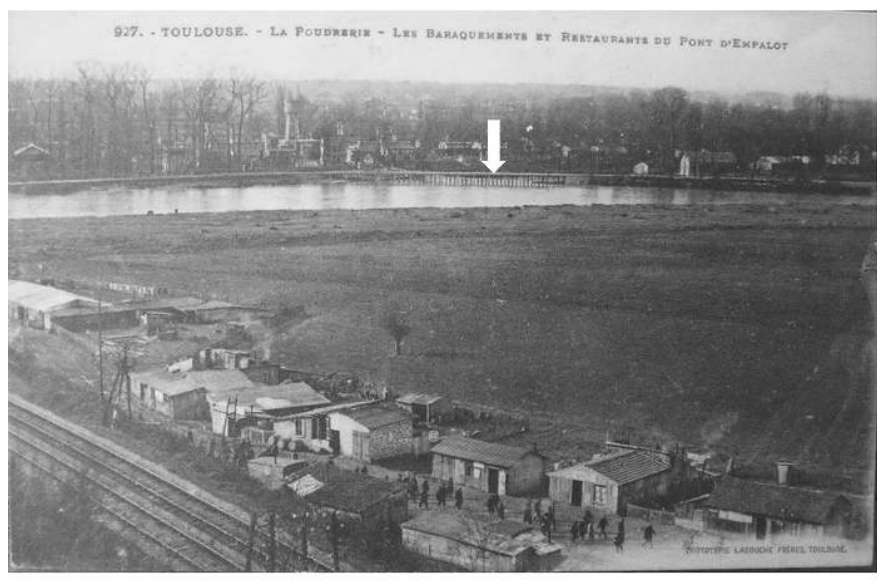

Début XX e siècle (Collection Ph Valette).

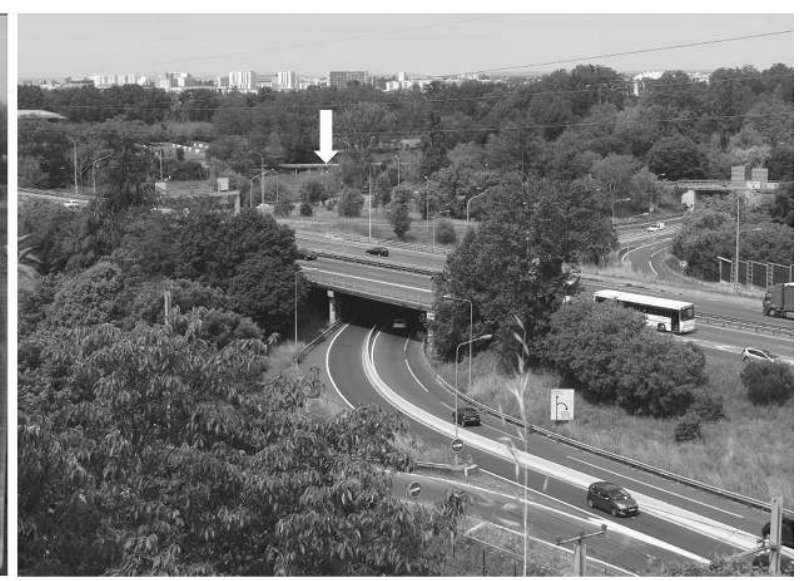

2013 (Ph Valette)

Figure 2 : L'urbanisation de la vallée de la Garonne : exemple d'Empalot au Sud de Toulouse The urbanization of the Garonne Valley: example of Empalot (south of Toulouse)

Entre les deux dates, à côté de la voie ferrée (en bas de l'image à gauche) vient s'adosser le périphérique toulousain et l'échangeur d'Empalot. Au-dessus du point de repère (flèche), l'urbanisation post années 1950 apparaît à travers le quartier de La Faourette avec ses barres d'immeubles d'habitat collectif.

Between the two dates, next to the railway (bottom image left) comes to lean the Toulouse device and exchanger Empalot. Above the benchmark (arrow), the post-1950 urbanization appears through the La Faourette with bars of collective housing buildings. 

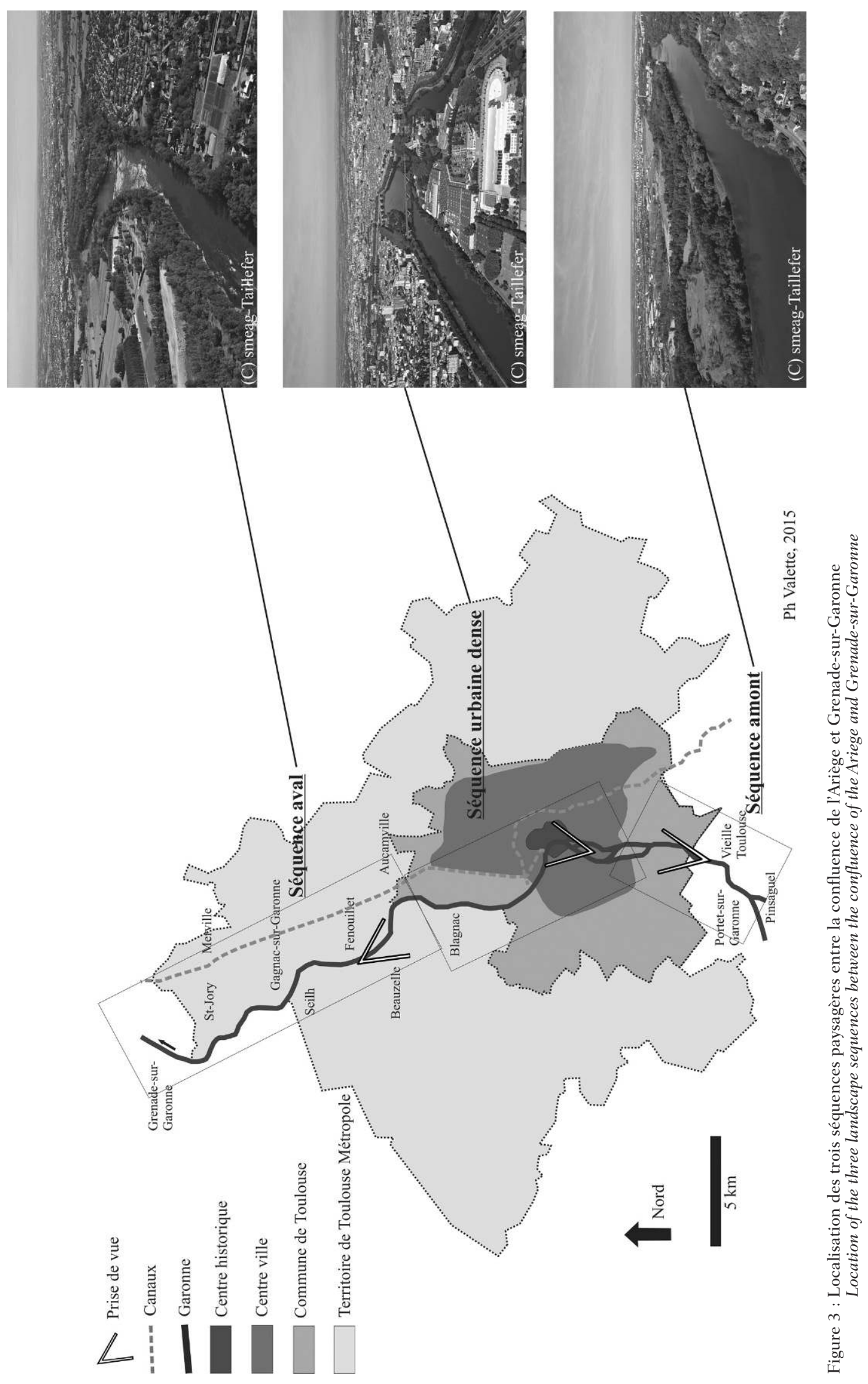
dense domine et partout la Garonne est artificialisée par de nombreux aménagements (digues, chaussées, ponts). Cette partie a été définie comme «l'arc culturel »(CUGT, 2012). Cette séquence paysagère est très minérale car densément urbanisée.

La troisième séquence est située à l'aval de la ville dense, entre Fenouillet et Grenade-sur-Garonne (figure 3). Les paysages se caractérisent par une dilution de l'urbanisation et une place de plus en plus forte des paysages agricoles, basée sur une agriculture intensive (céréaliculture et arboriculture irriguée). La Garonne vient buter régulièrement sur la rive gauche et offre une rive droite plus étendue, dans laquelle se trouvent les réseaux de communication (voie ferrée, canal latéral à la Garonne, route nationale et autoroute). L'agriculture est une composante prégnante de l'identité culturelle et paysagère de cette séquence, où apparaît encore l'ancienne activité de maraîchage autour de Blagnac.

\section{INTENTIONNALITÉS PAYSAGÈRES ET TEMPORALITÉ : RECONSTITUTIONS DE LA TRAJECTOIRE PAYSAGÈRE DES PAYSAGES FLUVIAUX URBAINS TOULOUSAINS}

Un regard géohistorique est nécessaire pour comprendre l'état actuel des paysages fluviaux urbains et périurbains toulousains (Jacob-Rousseau, 2009; Valette et Carozza, 2009). Cet état actuel, de Portetsur-Garonne jusqu'à Grenade-sur-Garonne, est le fruit d'une longue évolution, associée à des intentionnalités paysagères multiples. Dans ce contexte, l'utilisation de séries photographiques permet de saisir « d'un seul coup d'œil » les intentions d'aménagement des paysages au cours du temps.

\section{Une exploitation de la force hydraulique et une organisation des paysages disparate (de la période médiévale jusqu'au $\mathrm{XVII}^{\mathrm{e}}$ siècle)}

Les limites de la ville de Toulouse restent circonscrites à l'intérieur des remparts de la période médiévale jusqu'au XVII ${ }^{\mathrm{e}}$ siècle. Plusieurs faubourgs se développent comme celui de Saint-Cyprien en zone inondable ou celui de Saint-Michel sur la terrasse alluviale de rive droite (à l'abri des inondations).
À l'intérieur de la ville, la Garonne draine les richesses avec l'exploitation de la force hydraulique. Un quartier aux activités pré-industrielles est bien identifié autour de l'île Tounis (île à l'intérieur de la ville). Il faut associer à ce quartier trois complexes minotiers qui ont nécessité l'aménagement de chaussées (Bazacle, Daurade et Château Narbonnais). Accompagnant ces moulins, sept ports fluviaux pour charger et décharger les marchandises transportées par bateaux sont identifiés dans la ville (figure 4; Valette, Carozza, 2013). Enfin, des restes de piles de ponts émaillent le cours de la Garonne car durant cette époque aucun pont ne résiste aux crues et inondations.

Cette période se caractérise par peu de volontés de valoriser les paysages fluviaux urbains toulousains. Sur la rive droite, les maisons se présentent " dépourvues de défense et entièrement ouvertes sur le fleuve » (Laffont, 1997). Ces rangées de maisons, plus ou moins disparates s'interrompent pour faire face à des grèves ou des ports fluviaux. En face, sur la rive gauche, plus basse et soumise aux inondations, des hôpitaux et léproseries sont construits sur les berges, à l'écart du centre ancien hors d'eau.

Ailleurs en amont et en aval de Toulouse, les principaux bourgs restent limités en surface et se développent autour du fleuve. Portet-sur-Garonne, Blagnac, Fenouillet, Saint-Caprais, et Grenade-surGaronne sont construits à proximité du fleuve et bénéficient des apports de la navigation fluviale. L'étymologie de Portet est liée à l'activité portuaire fluviale. À cette époque, la Garonne est particulièrement instable et s'éloigne progressivement de tous ces centres villageois (Valette, Carozza, 2009). Aujourd'hui, au pied des centres urbains, nous trouvons encore la trace de l'ancien cours du fleuve. En dehors de ces bourgs, les paysages fluviaux sont agrémentés de ripisylves et de ramiers (peupleraies) dans les secteurs les plus bas et par des parcelles agricoles sur le palier le moins soumis aux crues. Ce schéma reste valable jusque dans la seconde moitié du $\mathrm{XX}^{\mathrm{e}}$ siècle pour ces territoires situés en-dehors de la ville (séquence amont et séquence aval).

Du Moyen Âge jusqu'au XvII ${ }^{e}$ siècle, on utilise l'eau de la Garonne pour différentes fonctions et les seules intentions d'aménagement des paysages consistent à valoriser et exploiter la force hydraulique (minoteries, navigation). Les premières intentionnalités de valorisation et d'embellissement des 


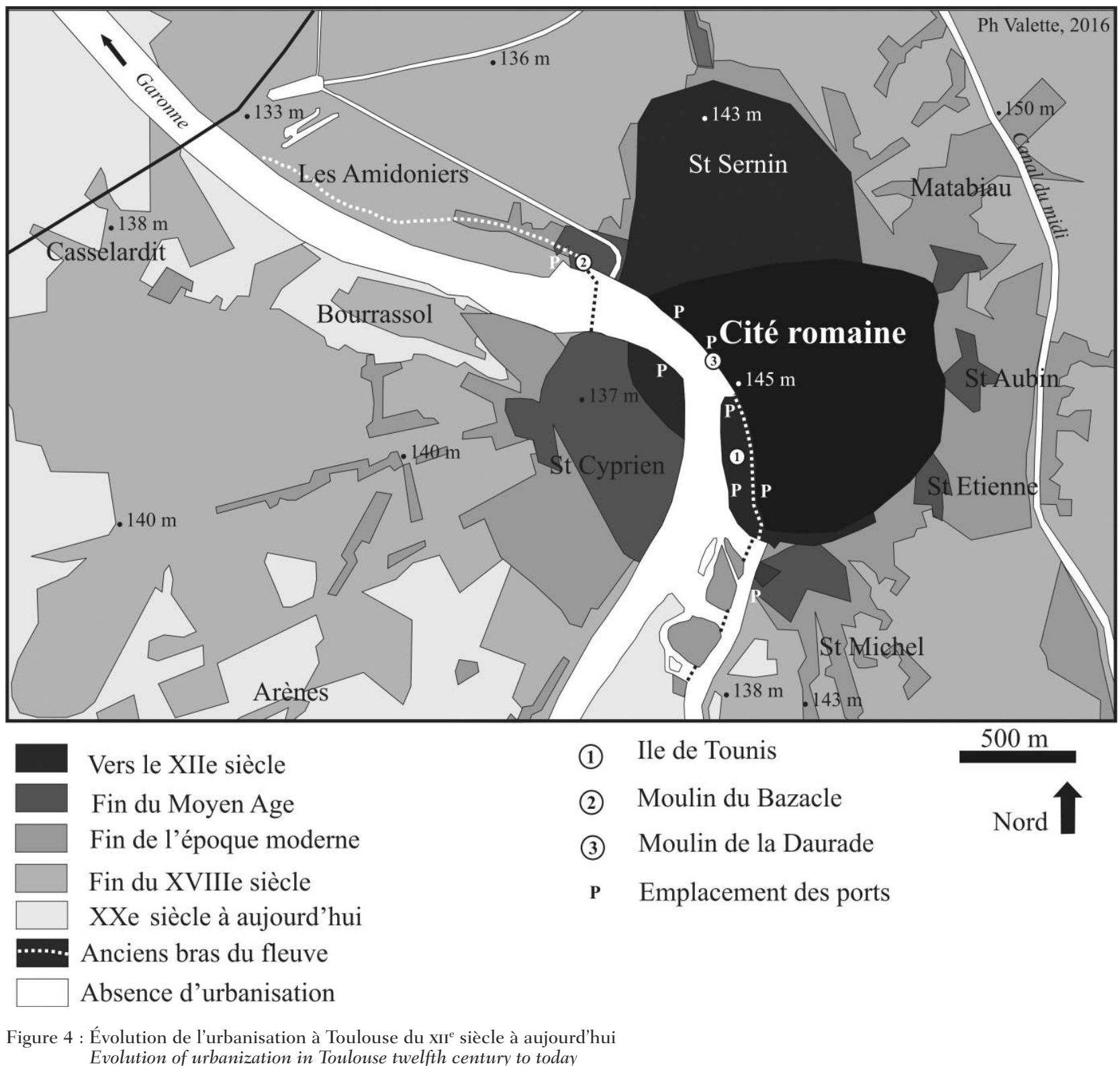

paysages apparaissent au XVII ${ }^{\mathrm{e}}$ siècle à l'intérieur de la ville. En dehors, en amont et en aval, seule compte l'exploitation de la force hydraulique.

\section{La lutte contre les inondations et la valorisation des paysages (du XVII ${ }^{\mathrm{e}}$ siècle jusqu'à la fin du XIX ${ }^{\mathrm{e}}$ siècle)}

Durant cette période, la plupart des aménagements vise à protéger la ville contre les crues et les inondations mais aussi à permettre la valorisation des paysages fluviaux urbains. Cette période longue de deux siècles, est à l'origine de la grande majorité des paysages fluviaux urbains que nous observons aujourd'hui.

En 1601, un remblai de $540 \mathrm{~m}$ de longueur est aménagé sur la rive gauche pour protéger le quartier Saint-Cyprien des inondations (Valette, Carozza, 2013). Ce remblai prend le nom de «Cours des Ormes » puisqu'il est planté de rangées d'arbres et a servi à partir de cette date d'espace de promenade pour les toulousains. À ses pieds, trois îles com- 
posent la future prairie des filtres, zone d'expansion des crues. En 1660, le pont neuf est terminé et il restera jusqu'au XIX ${ }^{\mathrm{e}}$ siècle (généralisation des ponts suspendus) le seul pont sur la Garonne. Il est aujourd'hui, une figure identitaire des paysages fluviaux urbains toulousains.

"Le XVIII ${ }^{\mathrm{e}}$ siècle est vraiment celui des grands travaux d'aménagement et de protection de la ville contre la Garonne » (Fortuné, 1988). Entre 1741 et 1789 , les quais et les ports sont réorganisés au sein de la ville. Leur fonction est double à la fois pour la navigation mais aussi pour canaliser les eaux de crues à l'intérieur du lit mineur. Des quais uniformes en brique rouge sont aménagés, dans lesquels les ports de la Daurade, de la Viguerie (Saint-Cyprien) et de Saint-Pierre sont réorganisés et embellis (figure 4). Sur la rive droite, au-dessus des quais sont aménagés des complexes immobiliers qui créent une vraie façade avec vue sur le fleuve. Sur la rive gauche plus basse, les maisons du quartier Saint-Cyprien sont protégées par une digue en brique. Plus tard, entre 1850 et 1866, le même modèle d'aménagement, alliant « l'utile et le beau » est repris au niveau de l'île Tounis en amont. De cette façon, à la fin du XIX ${ }^{\mathrm{e}}$ siècle, l'alignement des quais et des habitations sur la rive droite de la Garonne crée un nouveau paysage fluvial urbain, qui est devenu, lui aussi, un des paysages identitaires de la ville. L'observation de ces paysages à travers des photographies anciennes et actuelles montre que la trame de ces paysages n'a pas connu d'évolution majeure, si ce n'est au niveau des usages (figure 5). Ailleurs en amont et en aval, jusqu'au milieu du XIX ${ }^{\mathrm{e}}$ siècle, la vulnérabilité face aux risques d'inondation est un facteur qui limite l'urbanisation. Les premiers noyaux villageois se sont donc constitués à l'abri des crues sur la haute terrasse de la rive gauche; c'est le cas de Portet-sur-Garonne, Blagnac, Beauzelle, Seilh ou encore Grenade-sur-Garonne (figure 1). Tous ces bourgs ruraux entretiennent des relations avec le fleuve pour l'exploitation des riches terres agricoles ou pour le commerce (navigation). La rive droite est aussi habitée mais de deux manières. En zone inondable, nous trouvons Fenouillet, le hameau de Saint-Caprais, Ondes ainsi qu'une partie de Saint-Jory. Ces foyers de population se sont construits sur les paliers les plus hauts de la plaine inondable, de telle sorte que ces noyaux villageois sont protégés des crues les plus fréquentes. Mais cette localisation n'épargne pas ces villages des crues centennales, crues plus exceptionnelles. Par exemple, la crue de 1875 a particulièrement touché le village de Saint-Caprais, l'événement étant imprimé dans la mémoire collective grâce à la présence d'une plaque commémorant le niveau atteint par cette crue la plus haute connue dans le secteur à ce jour. Le positionnement au cœur du lit majeur permet néanmoins de développer une polyculture riche, jouant sur la complémentarité des terroirs, en suivant un gradient proximal-dis-

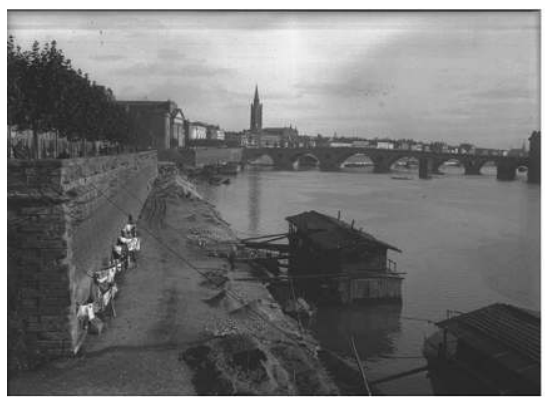

Fin XIX ${ }^{e}$ siècle (Museum d'histoire naturelle de Toulouse, Trutat)

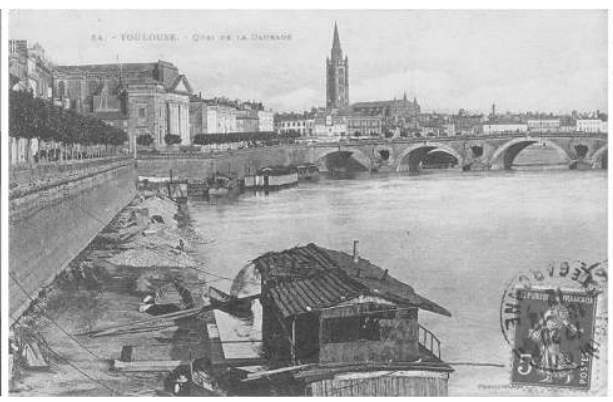

Début XXe siècle (Collection Ph. Valette)

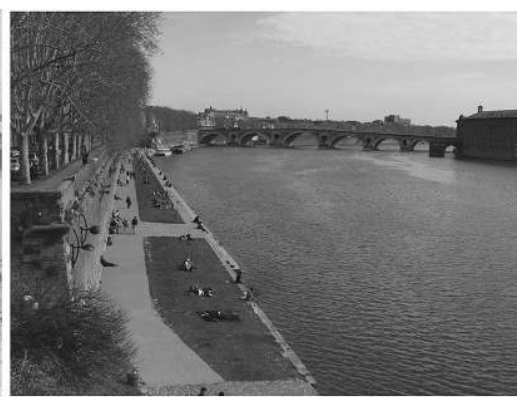

Avril 2011 (cl. Ph. Valette)

Figure 5 : Les paysages fluviaux urbains toulousains : les aménagements des quais du XvIII ${ }^{\mathrm{e}}$ siècle comme figure identitaire de la ville Urban fluvial landscapes of Toulouse: the amenities of the eighteenth century docks as identity figure of the city

Peu de changement dans les formes du paysage sont à noter, si ce n'est la disparition du clocher de la Dalbade. Les quais et ports de la rive gauche sont un espace de travail (lavandières, extracteurs de sables et graviers, restes de batellerie) jusqu'au milieu du xx siècle. Depuis les années 1990, ils sont devenus un espace de promenade et de flânerie.

Little change in the forms of landscape are noteworthy, if not the disappearance of the bell tower of the Dalbade. The docks and ports on the left bank are a workspace (washerwomen, sand and gravel extractors, skippers scraps) until the mid-twentieth century. Since the 1990s, they became a space for walking and strolling. 
tal avec le fleuve. La Garonne offre des ressources halieutiques, le lit majeur immédiat est occupé par des prairies humides, des peupleraies, des saulaies (ramiers), et du maraîchage, alors que l'on cultive des céréales, fruits, légumes et vignes dans le paysage de bocage du lit majeur exceptionnel. Plus en hauteur, à l'abri des crues du fleuve nous trouvons Vieille-Toulouse et Saint-Jory.

Les intentionnalités paysagères du XVII jusqu'à la fin du XIX siècle sont multiples. Dans le centre ancien, il s'agit d'abord de lutter contre les inondations, tout en embellissant la ville. Les paysages fluviaux urbains en gardent encore la trace, à tel point qu'ils font partie des paysages identitaires aujourd'hui. Dans les zones périurbaines, l'intentionnalité est de favoriser et de développer l'agriculture en adoptant une polyculture (céréales, marâ̂chage, vignes, prairies, peupleraie).

\section{Du développement de loisirs nautiques à la dégradation progressive des paysages fluviaux (de la fin $\mathrm{XIX}^{\mathrm{e}}$ siècle aux années 1990)}

Cette période se caractérise par une dualité et une progressivité dans les intentions paysagères au sein du centre ancien. D'un côté, plusieurs usages ludiques se développent : nautisme sur le bras principal du fleuve, baignade et promenade dans le parc toulousain sur l'île du Ramier. Néanmoins, c'est aussi une période d'abandon progressif du fleuve. La construction du canal latéral, et surtout l'arrivée du rail, mettent un terme définitif à la navigation fluviale durant le XIX ${ }^{\mathrm{e}}$ siècle (Valette, 2002). Ainsi, le centre de gravité économique se déplace vers le lit majeur, puis sur les terrasses alluviales. La Garonne est alors progressivement abandonnée et se replie sur elle-même. L'ensemble des paysages fluviaux garonnais connaît un mouvement d'obsolescence initié par l'abandon du commerce sur la voie d'eau. La conséquence se traduit par une dégradation progressive des paysages qui connaît sa phase la plus intense après les années 1950.

Jusque dans la seconde moitié du $\mathrm{Xx}^{\mathrm{e}}$ siècle, la ville de Toulouse et les villages voisins se développent en surface mais restent relativement regroupés autour du noyau ancien. Après la Seconde Guerre mondiale, l'urbanisation et la périurbanisation gagnent les espaces contigus à la ville de Toulouse et ceux entre les noyaux villageois anciens. Face à ce mouvement, à partir des années 1950-1960, la société riveraine met en place un système de digues en béton en amont de la ville (Empalot) et en aval (jusqu'à Blagnac, figure 1). Cependant, les matériaux utilisés et la hauteur des ouvrages viennent totalement en rupture avec les aménagements précédents. Aucune intentionnalité visant à valoriser les paysages n'a présidé à ces aménagements et le fleuve suscite peu d'intérêt. D'ailleurs les accès aux berges sont très limités. L'intention est véritablement de maîtriser le fleuve. Ces digues créent de nouveaux paysages en supprimant de nombreux moulins qui ont construit pendant longtemps l'identité de la ville (figure 6). À Blagnac, le moulin est également transformé ainsi que tous ses abords : asséchement du bras, aménagement du stade (figure 6). Ailleurs dans la ville, certains bras sont asséchés comme au niveau de la Garonnette (figure 7) ou au Bazacle. Durant cette époque, l'île du Ramier au sud, qui pendant longtemps a abrité la poudrerie (activité industrielle) et le parc toulousain (espace de promenade et loisirs), est dotée de différents équipements (stade, piscine, parc des expositions...).

Cette période de dégradation progressive des paysages fluviaux s'étend également en amont et en aval de la ville de Toulouse. La plaine inondable continue d'être exploitée par l'agriculture, qui devient dans la première partie du $\mathrm{xx}^{\mathrm{e}}$ siècle l'activité essentielle. Après la Seconde Guerre mondiale, l'agriculture du lit majeur, comme partout dans d'autres régions françaises, connaît de profondes mutations (remembrement, monoculture, mécanisation, irrigation) tandis que les activités industrielles se polarisent autour des grands axes de transports, au-delà du lit majeur. À l'amont comme à l'aval de Toulouse, le paysage fluvial se crible de gravières, d'abord en lit mineur puis ensuite en lit majeur. À l'intérieur de ces gravières sont extraits les matériaux nécessaires à la construction des différentes infrastructures urbaines. D'autre part, malgré leur soumission au risque d'inondation, les paysages fluviaux situés en amont et en aval de Toulouse n'échappent pas à la périurbanisation. Toutefois, les communes situées au cœur de la plaine inondable accusent un léger retard d'urbanisation, si on les compare aux espaces aux marges du lit majeur à la fois sur la rive droite et la rive gauche. 


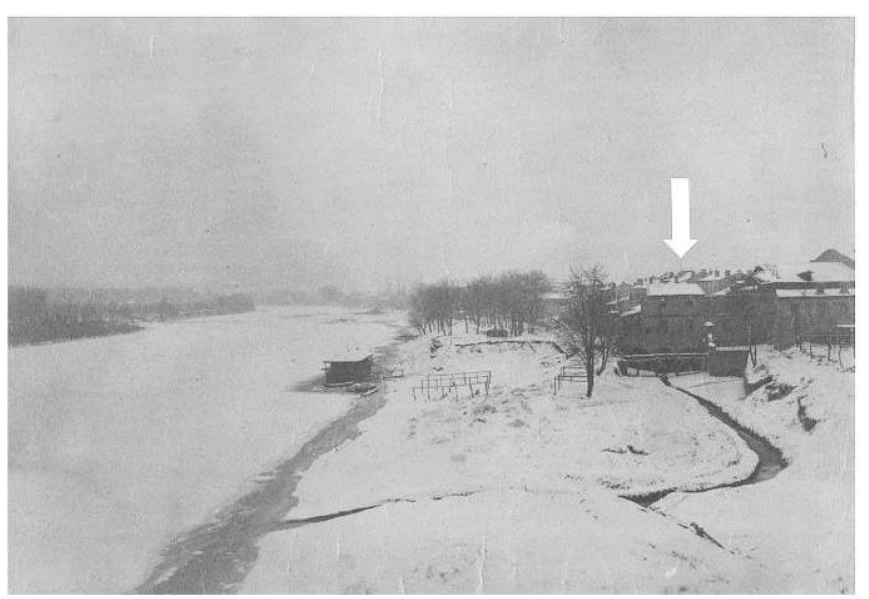

Fin XIXe siècle (Muséum d'Histoire Naturelle de Toulouse, Trutat)

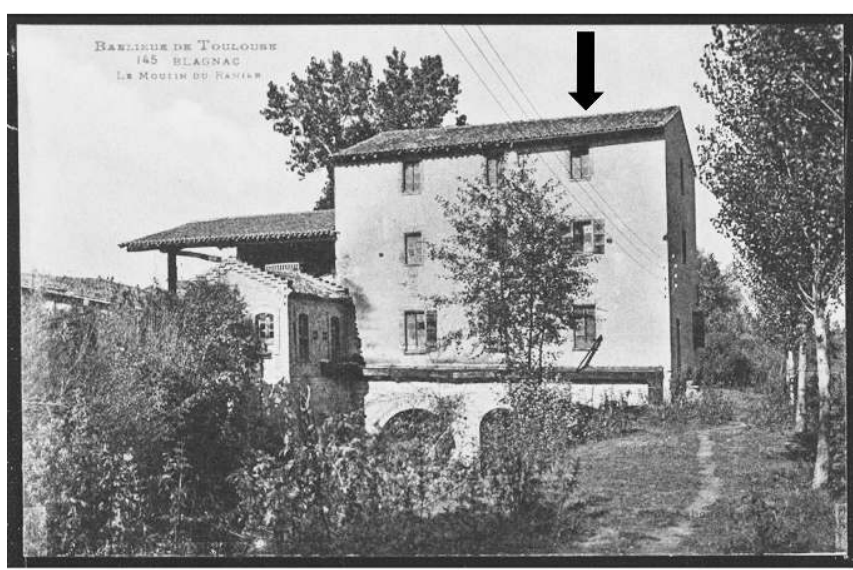

Début XX e siècle (Collection Ph Valette)

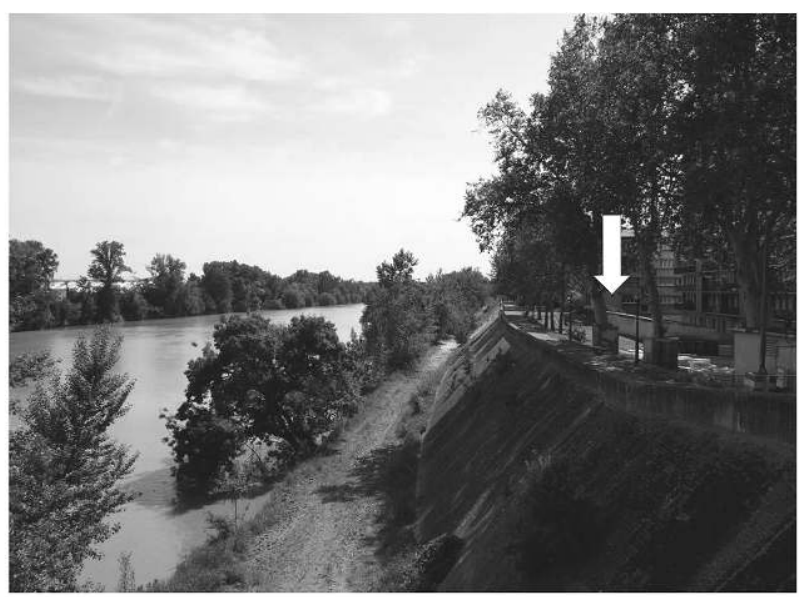

Juin 2013 (Philippe Valette)

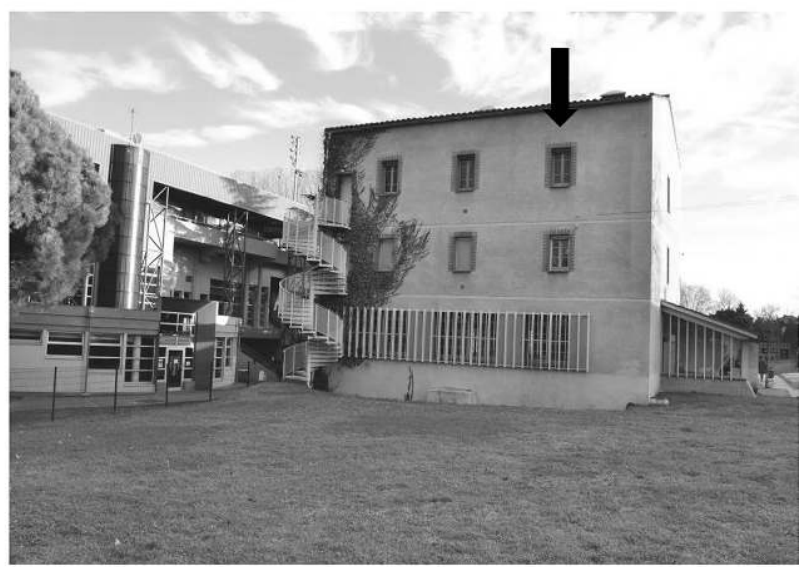

Février 2014 (Causel, Bosque)

Figure 6 : Disparition des moulins : exemple du moulin en amont du pont Saint-Michel (en haut) et du moulin de Blagnac (en bas) Disappearance mills : example of Saint-Michel bridge mill (top) and Blagnac mill (bottom)

Certains édifices disparaissent des paysages comme en amont du pont Saint-Michel et sont remplacés par des digues. D’autres sont transformés comme à Blagnac. Ces deux exemples montrent une véritable métamorphose des paysages fluviaux.

Some buildings are disappearing landscapes as upstream of Saint-Michel Bridge and are replaced by dikes. Others are processed as in Blagnac. These two examples show a metamorphosis of river landscapes.

\section{Un retour au fleuve dès les années 1990 en ville}

À partir des années 1990, la municipalité de la ville de Toulouse met en place un plan Garonne dont « les objectifs sont la valorisation des paysages, la protection des équilibres naturels et la mise en place d'espaces verts » (Centre Urbain d'Initiation à l'Environnement de Toulouse, CUIET, 1993). Entre les années 1990 et 2000, ce plan se traduit par la multiplication d'initiatives où l'intentionnalité paysagère se focalise sur la valorisation des paysages fluviaux : mise en place de cheminements doux au sein de la ville sur les berges, suppression des parkings à voiture (figure 8), reconversion de bâtiments et édifices (château d'eau, abattoirs, théâtre Garonne, manufacture des tabacs...), aménagements d'espaces verts (prairie des Filtres, jardin Raymond VI...). Au cours des dernières décennies, beaucoup d'initiatives se sont appuyées sur la mise en valeur architecturale et culturelle des berges en ville. Les digues en bétons sont valorisées, plantées de rangées d'arbres, agrémentées de bancs et servent de lieux de promenade. Autre initiative symbolique, la végétalisation des digues est tentée mais échoue durablement. 


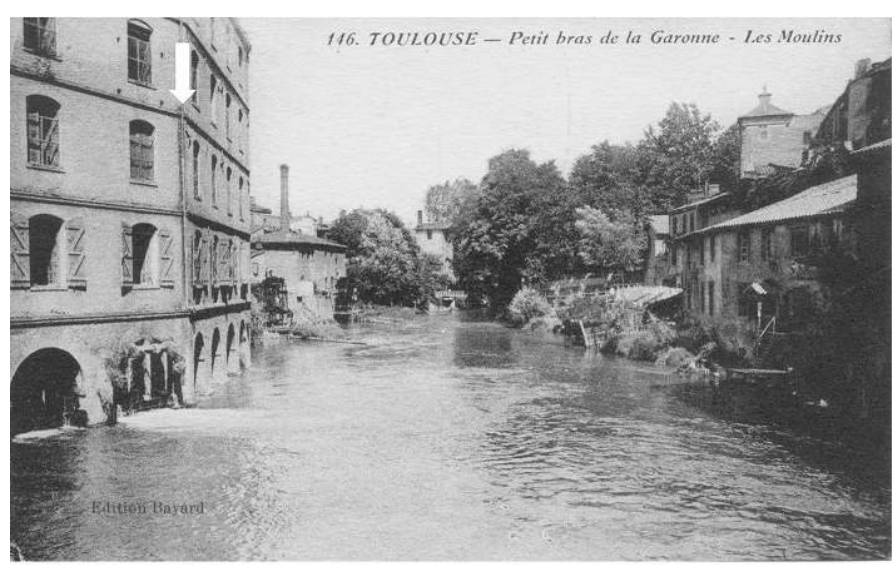

Début XX e siècle (Collection Ph Valette)

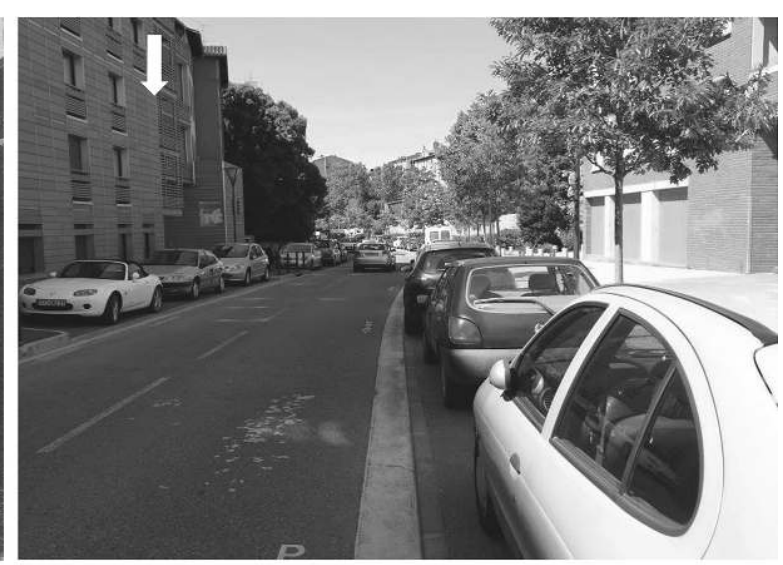

Juin 2013 (Philippe Valette)

Figure 7 : Évolution de la Garonnette : d'un quartier pré-industriel à une rue asséchée Evolution of the Garonnette: a pre-industrial district to a dried Street

Les anciens moulins du bras secondaire ont laissé la place à des immeubles d’habitation. La Garonnette a été asséchée en 1950. Elle laisse place à une rue bordée de parkings à voitures.

The old mills of the secondary arm gave way to apartment buildings. Garonnette was drained in 1950. It leaves room for a street lined with car parking.

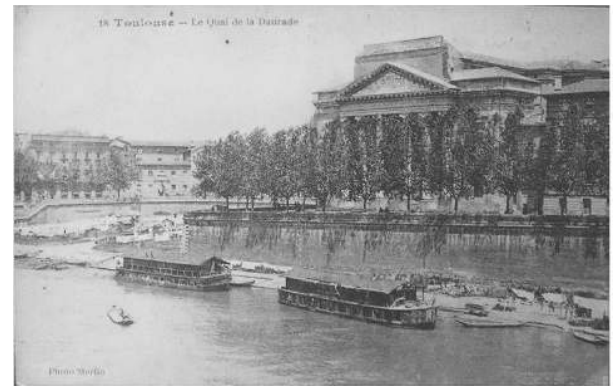

Début XXe siècle (Collection Ph. Valette)

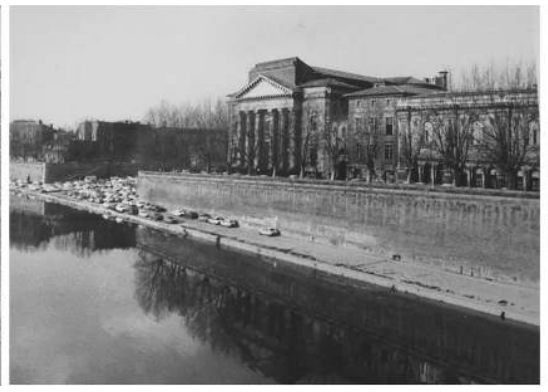

Années 1960 (Collection Ph. Valette)

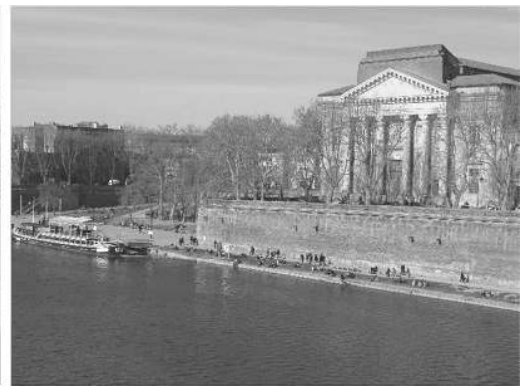

Mars 2012 (cl. Ph. Valette)

Figure 8 : Les quais de la Daurade à Toulouse The banks of the Daurade Toulouse

Chacune des photographies témoigne d'usages différents : lavandières (début xx ${ }^{\mathrm{e}}$ siècle), parking à voiture (années 1960) et lieu de promenade (aujourd'hui).

Each photograph shows various uses : washerwomen (early twentieth century), car park (1960) and place of promenade (today).

Toutes ces initiatives, jusque dans les années 2000, ont visé à remettre la Garonne au cœur du centre-ville. Mais, ce renouveau est loin d'atteindre l'envergure de certains projets en France, comme celui de Bordeaux par exemple. Pourtant la demande paysagère contemporaine est considérable. Le terme de paysage a envahi toutes les sphères de la société. Il devient un objet de politiques publiques et les intentionnalités visées sont celles de son amélioration et sa valorisation. Certains auteurs parlent d'empaysagement (Debarbieux, 2007). « La face visible de l'empaysagement désigne [...] un tournant dans la façon qu’ont les sociétés contemporaines de se penser elles-mêmes et de penser leur inscription matérielle par l'entremise de la représentation et de l'action paysagère » (Debarbieux, 2007). Ce dernier peut être considéré comme une forme de banalisation de l'invocation du paysage et du souci paysager dans toutes les formes d'aménagement.

Au final, notre regard géohistorique nous permet de distinguer plusieurs types d'intentionnalités au cours du temps à l'origine des paysages fluviaux actuels (figure 9). Parmi ces dernières, l'intentionnalité d'embellissement des paysages occupe une grande part aujourd'hui. Dans le passé, elle s'est focalisée sur le centre ancien toulousain. Aujourd'hui, l'ambition du projet de « Grand parc Garonne », initié depuis les années 2010 est bien de 


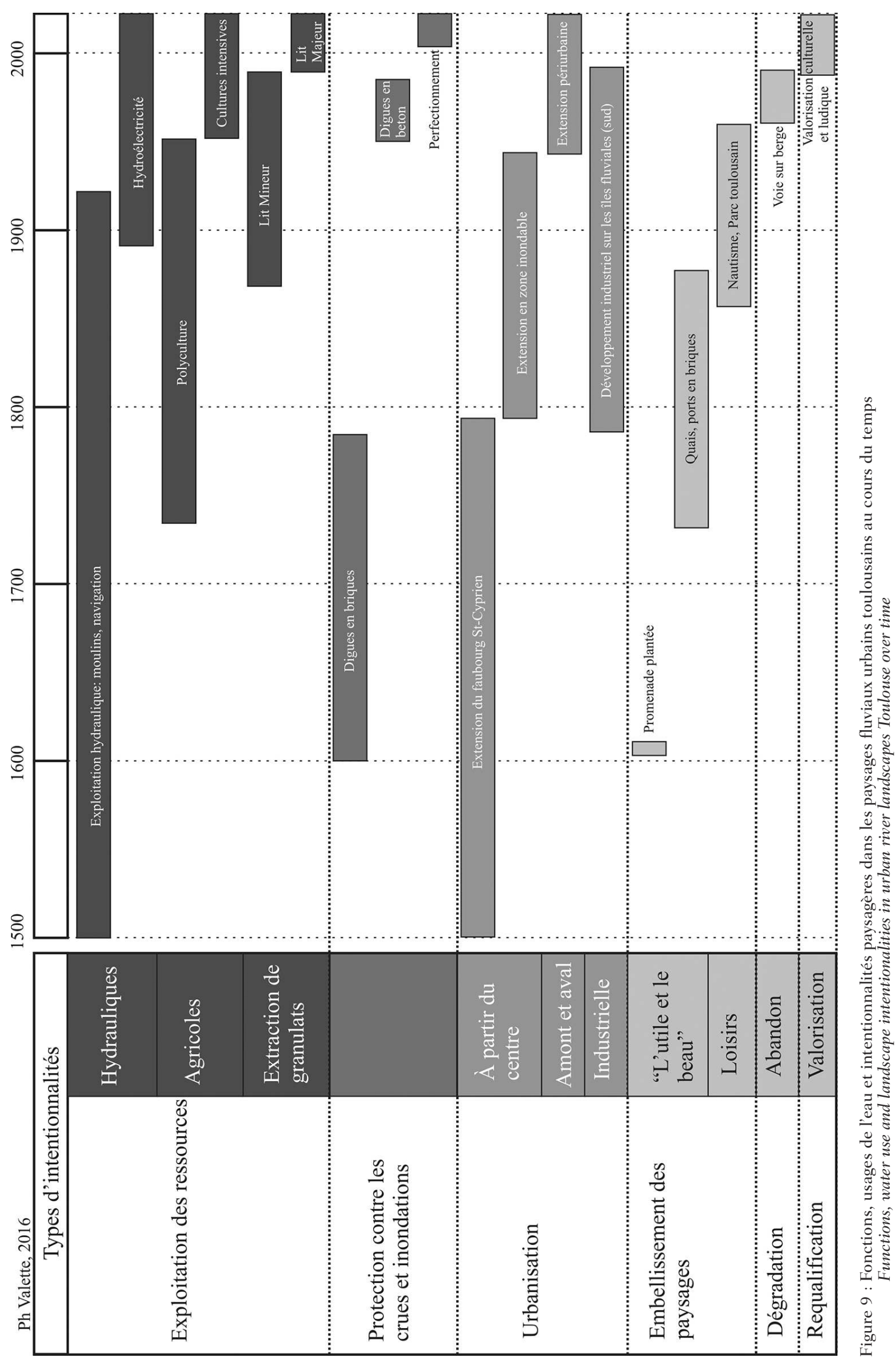


reconquérir et valoriser la Garonne sur un territoire plus vaste que le seul centre ancien en intégrant les communes périurbaines voisines. Qu'en est-il exactement de ces espaces périurbains en amont et aval de la ville? L'intentionnalité paysagère visant la valorisation et l'embellissement des paysages fluviaux, visible dans le centre ancien, se diffuse-t-elle sur ses marges?

\section{DIFFUSION DU MODÈLE D'INTENTIONNALITÉ D'EMBELLISSEMENT DES PAYSAGES FLUVIAUX AU NORD ET AU SUD DE LA VILLE}

Sur les territoires situés en amont et aval de la ville de Toulouse, il est possible de repérer des signes de valorisation des paysages fluviaux qui tendent à aller dans le sens d'une diffusion de l'intentionnalité paysagère du centre ancien. Par contre, d'autres signes, au contraire montrent l'inverse.

\section{Vers une «balconisation" des paysages fluviaux?}

La redécouverte des berges au centre de Toulouse est marquée par une volonté d'améliorer la visibi- lité sur la Garonne. En 2006, un belvédère est créé au niveau du jardin Raymond-VI où l'espace public s'ouvre sur le fleuve. Telle une terrasse, ce belvédère s'avance au-dessus de l'eau, en enjambant la haute digue de béton, pour offrir un panorama sur la chaussée du Bazacle et le centre historique. Utilisés pour ne pas occulter la vue, les gardes corps à barreau rappellent ceux des nouveaux balcons privés sur la Garonne. Un peu plus tard en 2008, une passerelle est ouverte entre le port de la Viguerie et le jardin Raymond-VI afin de permettre la continuité pédestre sur cette rive gauche.

La redécouverte du fleuve comme aménité paysagère à Toulouse a eu des conséquences très marquées sur les dynamiques immobilières. Depuis une vingtaine d'années, les immeubles de standing proposant des appartements avec vue sur l'eau se multiplient, remplissant les espaces laissés vacants par les anciennes industries. Ces complexes immobiliers se situent à proximité de l'ancien port Garaud (figure 10), sur l'ancienne île du Bazacle et à proximité des anciens abattoirs. Une tendance qui s'est accentuée ces cinq dernières années et qui répond aux aspirations d'une classe sociale aisée, qui n’hésite pas à payer au prix fort le luxe d'un panorama sur la Garonne. En 2011, les anciennes papeteries Job situées à l'aval du Bazacle sont réhabilitées en

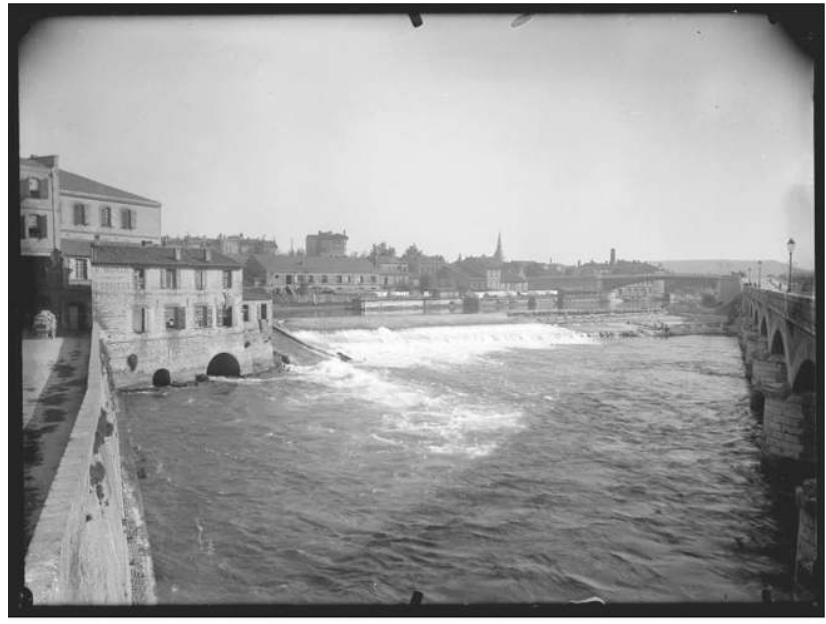

Fin XIXe siècle (Muséum d'Histoire Naturelle de Toulouse, Trutat).

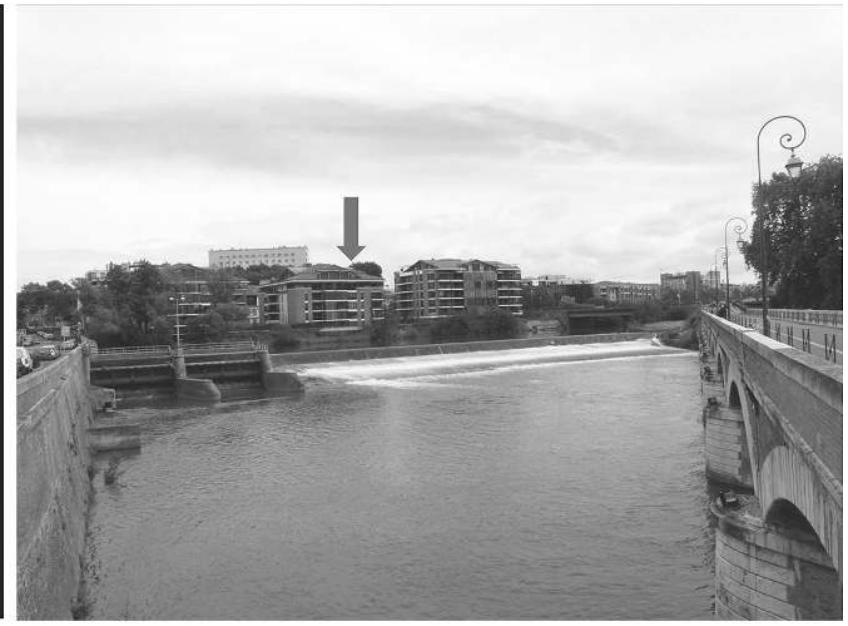

Mai 2011 (Giral)

Figure 10 : Balconisation des paysages de la Garonne à Toulouse Balconisation landscapes of the Garonne in Toulouse

Depuis quelques années, de nombreux immeubles sont construits sur les berges de la Garonne, en marge du centre ancien. Les balcons des appartements offrent une vue imprenable sur le fleuve.

In recent years, many buildings are built on the banks of the Garonne, on the fringes of the old center. The balconies of the apartments offer stunning views of the river. 
appartements. Ici, les façades blanches, arrondies, et les grands balcons donnent au paysage fluvial urbain un air de front de mer. Cette «balconisation » des paysages fluviaux urbains s'étend à l'amont et l'aval immédiat du centre ancien (Valette et al, 2013).

Dans les communes périurbaines à l'aval et en amont, les panoramas sur le fleuve sont peu nombreux; les ramiers qui s'étendent au pied des communes tiennent la Garonne à l'écart des villages. D'autre part, la ripisylve, quasi continue sur les berges depuis la fin des digues toulousaines, fait office d'écran visuel. Les vues privatives sur l'eau sont très rares, tout comme les terrains constructibles au bord des falaises de la rive gauche. Les quelques panoramas existant depuis des espaces publics ne sont pas du tout mis en valeur, et les meilleurs indicateurs de cette indifférence ce sont les bancs, qui tournent allègrement le dos à la Garonne (comme à Beauzelle).

L'exemple du parc le plus récent, achevé en 2007 et le plus proche de la ville de Toulouse est révélateur de cet oubli de la Garonne. Dans un ancien ramier au contact du cœur historique de Blagnac, l'espace vert répond aux attentes actuelles en matière de parc urbain de qualité, dans une ville accueillant de nombreux cadres. Pourtant, depuis le sommet de la digue qui longe le fleuve, ce dernier est invisible, caché derrière une ripisylve dense. Le belvédère, en point d'orgue, ne s'élève pas au-dessus des obstacles visuels, de sorte qu'il ne permet d'observer que l'intérieur du ramier. Dans ce parc, l'aménagement paysager a remplacé l'ouverture sur le paysage fluvial (figure 11).

\section{Vers une Garonne comme espace de nature et de loisirs?}

Depuis une quinzaine d'années, la Garonne dans le centre de Toulouse a été dotée du statut d'espace public à vocation de loisirs et de détente pour les citadins. Ce territoire a pris le nom d'arc culturel dans le projet «Grand parc Garonne ». Sur l'Ile du Ramier, au sud, sont regroupés les terrains de sport alors que des activités nautiques animent le lit mineur (club d'aviron). Une partie du patrimoine bâti ancien en bord de Garonne a été réhabilité et les berges de la Garonne sont utilisées ponctuellement comme des lieux d'exposition temporaires ou des salles de spectacle à ciel ouvert (festival Rio Loco...).

Dans les territoires périurbains, la vocation des bords de la Garonne comme espace de loisirs et de nature a bénéficié d'opportunités. Leur présence est le reflet d'une forte naturalité anthropique héritée. Dans de nombreux endroits, l'extraction de granulats intensive en lit majeur laisse apparaître de nombreux trous d'eau qui ont été réhabilité en espace vert. En amont de Toulouse, la confluence de l'Ariège et de la Garonne est criblée d'anciennes fosses d'extraction. Sur ce site très anthropisé et dégradé est créé en 1983 le «parc naturel du confluent » (AriègeGaronne, figure 12) par la commune de Portet-surGaronne. En aval, à Beauzelle (ramier des Quinze

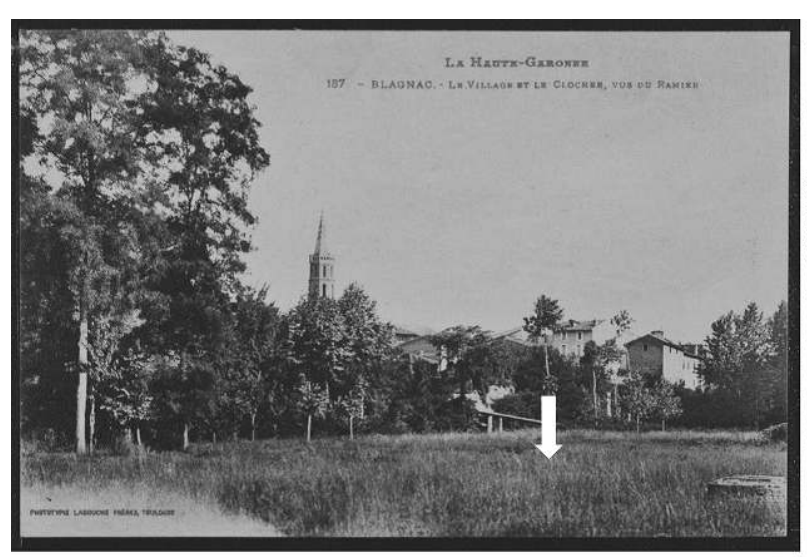

Début XXe siècle (Collection Ph Valette).

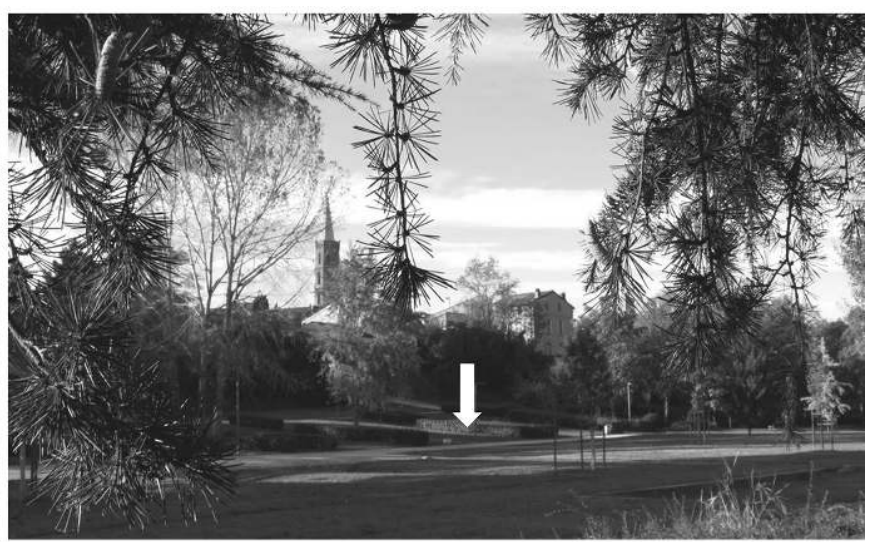

2013 (Mendizabal)

Figure 11 : Le parc de Blagnac protégé par une digue ne dialogue pas avec la Garonne The Blagnac park protected by a breakwater do not dialogue with the Garonne

Au début du $\mathrm{Xx}^{\mathrm{e}}$ siècle, la prairie ouverte sur la Garonne (située à droite et peu visible) est aménagée en parc urbain à partir de 2007. In the early twentieth century, the open prairie on the Garonne (to the right and inconspicuous) is converted into an urban park from 2007. 


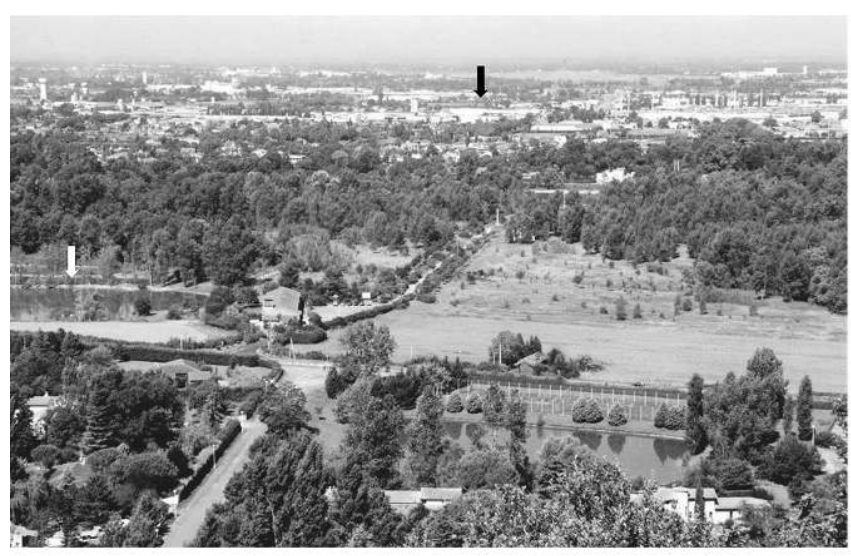

2003 (Nars)

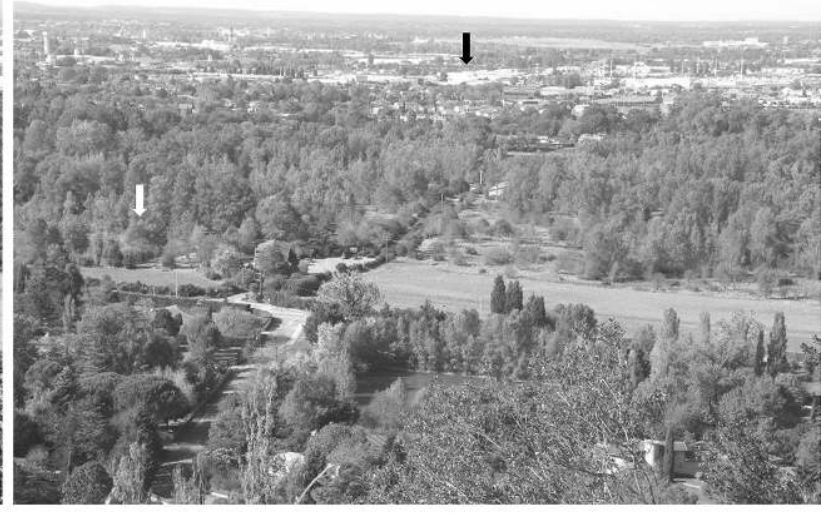

2013 (Bompard)

Figure 12: Le parc du confluent entre 2003 et 2013

The park confluence between 2003 and 2013

Entre les deux dates les anciennes fosses d'extraction de granulats sont masquées par la densification de la végétation. Au dernier plan se trouve la vaste zone d'urbanisation et de périurbanisation de Portet-sur-Garonne.

Between the two dates the old pits extraction of aggregates are masked by the densification of the vegetation. In the background is the vast area of urbanization and suburbanization of Portet-sur-Garonne.

sols) ou à Fenouillet, les bords de la Garonne ont fait l'objet de mise en place d'espaces verts et de loisirs par les différentes communes. Les ramiers sont trop souvent inondés pour être constructibles, d'autant qu'ils ne sont majoritairement pas protégés par des digues. À proximité immédiate des centres villes, ils représentent alors une réserve foncière qui ne peut être valorisée que par l'agriculture, ou par la création d'espaces de «nature » et surtout de loisirs.

Aujourd'hui le parc du confluent Ariège-Garonne est englobé dans un territoire plus vaste qui vient d'être labellisé Réserve Naturelle Régionale - Confluences Garonne Ariège (4 juin 2015). L'ouverture de ces parcs urbains en bord de Garonne a prévalu dans tous les territoires en amont et aval de Toulouse dès les années 1980, plus par effet d'aubaine que par une réelle intentionnalité de renouer avec le fleuve. Depuis les vingt dernières années, chaque commune aménage son espace de loisirs sans concertation avec les territoires voisins, de sorte que chacun d'entre eux semble déconnecté les uns avec les autres.

\section{Vers une continuité des parcours le long de la Garonne?}

La mise en place d'un cheminement ininterrompu le long des berges est un objectif affiché du projet actuel «Grand parc Garonne » (CUGT, 2012).
Le sommet des digues, construites dans les années 1960, à l'amont et l'aval immédiat du centre historique est réservé aux piétons/cycles. Elles sont depuis quelques années végétalisées (rangée de platanes pour créer de l'ombre et lierre pour atténuer la froideur des pentes en béton). Entre les deux, les quais de brique du XvIII ${ }^{\mathrm{e}}$ siècle retrouvent leur vocation d'espace de déambulation.

Mais le cheminement le long de la Garonne s'interrompt par endroits au cœur de la ville, particulièrement en rive gauche, où de nombreux bâtiments viennent «plonger dans le fleuve » (Hôtel Dieu Saint-Jacques). La quête de la continuité a conduit à construire, en 2008, une passerelle piétonne au-dessus de la Garonne pour relier le Jardin Raymond VI au quai de la Viguerie. Cette passerelle s'appuie sur la façade de l'un des monuments les plus emblématiques de Toulouse (Hôpital de la Grave).

Dans les territoires périurbains, des cheminements le long de la Garonne ou en hauteur (coteaux de Pech David) existent mais à mesure que l'on s'éloigne du centre-ville, ils deviennent de plus en plus étroits, et de moins en moins entretenus. Par exemple pour la partie en aval, les cheminements ne sont plus continus après une dizaine de kilomètres en rive droite, et seulement cinq en rive gauche. En amont, peu de chemins existent si ce n'est à proximité de Portet-sur-Garonne le long de la Garonne, 
ou sur les coteaux de Pech David. Cette réalité nous montre que des efforts doivent encore être réalisés pour satisfaire la continuité des parcours doux le long du fleuve.

Néanmoins, certains d'entre eux permettent de relier les parcs des ramiers (Blagnac et Beauzelle par exemple). Ils sont malheureusement peu visibles, peu accessibles, à mesure que l'on s'éloigne des centres villes. Beaucoup des habitants des communes périurbaines ignorent la possibilité de pouvoir rejoindre Toulouse en longeant la Garonne. Des efforts restent à fournir pour valoriser ces continuités dans les paysages périurbains, où les communes se contentent encore d'une gestion à minima. Ailleurs dans les vallées affluentes, comme celle du Touch, la présence du cours d'eau a permis la mise en place d'une coulée verte (Béringuier et al., 2014).

Des réflexions s'amorcent également sur la traversée du fleuve. En aval de Toulouse, où les rares ponts ne sont pas adaptés aux piétons/cycles, il n'est pas d'actualité d'investir dans la réalisation d'ouvrages réservés aux circulations douces. Mais, dans le cadre du projet "Grand parc Garonne », on s'intéresse à l'utilisation d'une passerelle déjà existante, qui reliait deux gravières à l'aval du pont de Gagnac-sur-Garonne. En amont, le bac de Portetsur-Garonne a repris du service depuis une dizaine d'années et permet de relier le centre du bourg au parc du confluent (figure 13). En définitive, si des projets sont évoqués, les réflexions sur les continuités et traversées sont bien loin encore d'une phase opérationnelle.

\section{Vers une Garonne délaissée et désaffectée?}

Entre l'objectif affiché de retour au fleuve sur le vaste territoire de Toulouse Métropole et la réalité des paysages, de nombreux décalages apparaissent aujourd'hui. Dans plusieurs endroits, les paysages fluviaux garonnais concentrent quelques nuisances et les paysages y sont désaffectés et délaissés. La rive droite à l'aval de Toulouse en est un bel exemple notamment dans le quartier Ginestous-Sesquières où les camps des gens du voyage côtoient espaces industriels et lotissements. Ici, les paysages fluviaux ne font l'objet d'aucune intentionnalité de valorisation et d'embellissement. Le fleuve y est souvent inaccessible. Plus en aval encore, à Fenouillet, les espaces en bord de fleuve sont abandonnés et désaffectés (ancienne piste de karting). À proximité, les sentiers qui longent la Garonne sont devenus inaccessibles. En face de cet espace se trouve le ramier des Quinze Sols, aménagé et valorisé en espace vert. Cette opposition forte d'une berge à l'autre témoigne de politiques d'aménagements réalisées au coup par coup sans réelle cohérence d'ensemble par chacune des communes (Beauzelle, Fenouillet). Sur la rive

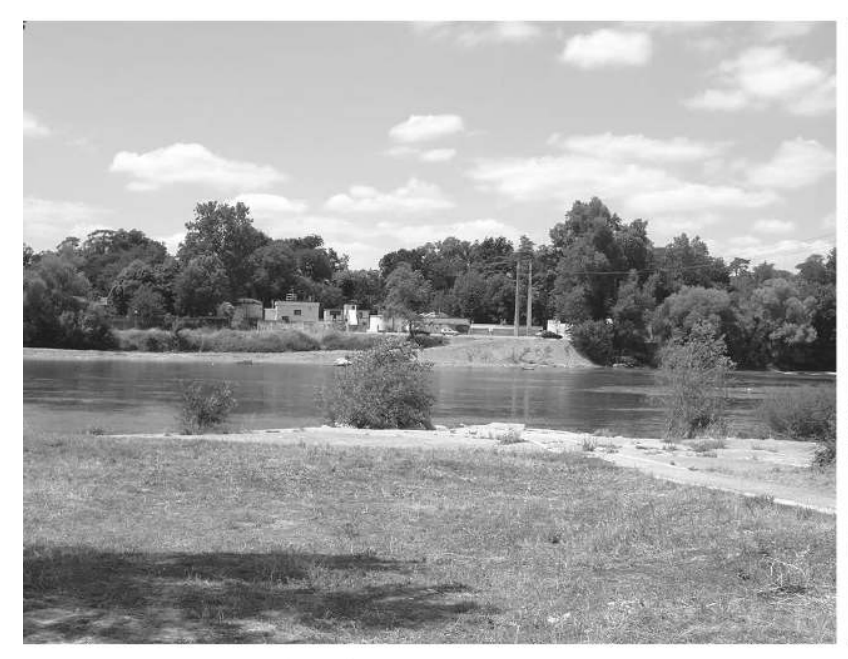

2005 (Nars)

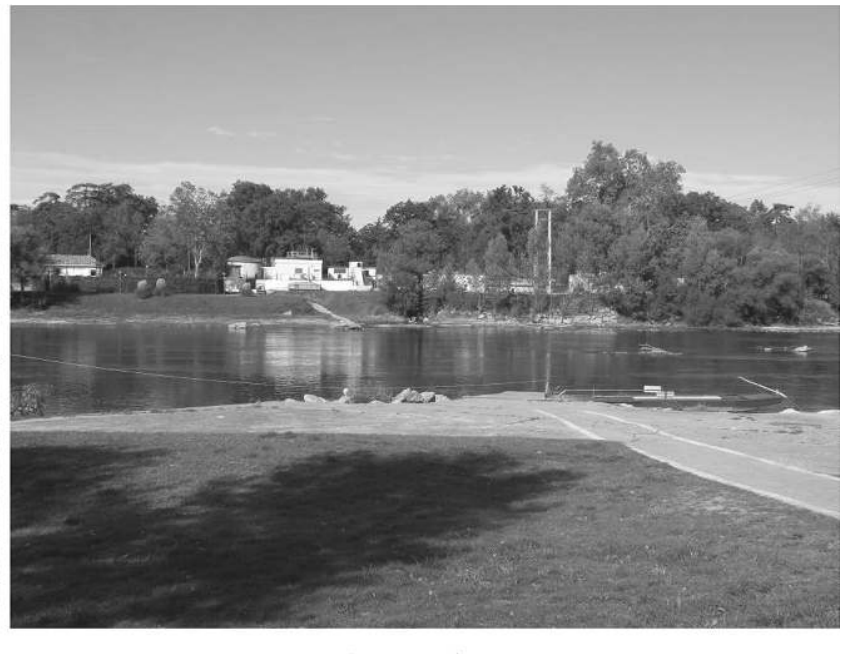

2013 (Bompard)

Figure 13 : Le bac de Portet remis en fonctionnement depuis 2006 permet la liaison entre le village (deuxième plan) et le parc du confluent (premier plan)

park (foreground)

The Portet tray back into operation since 2006 allows the connection between the village (second level) and the confluence of the 
gauche, quand la ripisylve s'ouvre, le spectacle de la rive opposée à Fenouillet témoigne du peu de cohérence dans la mise en valeur des points de vue sur le fleuve. À l'inverse sur la rive désaffectée vers le ramier des Quinze Sols, le point de vue est remarquable. De la même manière, les rares points de vue à partir de l'espace vert au pied de Blagnac débouchent sur une rive droite très peu valorisée.

En amont de Toulouse, les anciennes îles de la Garonne avaient une vocation essentiellement industrielle (poudrerie...). Cette activité a migré vers le sud de la ville à mesure que l'urbanisation s'étendait. L'explosion AZF en 2001 a provoqué une mutation des paysages avec la mise en place de l'oncopôle (campus de cancérologie) et la désaffection de nombreuses parcelles (figure 14). Par ailleurs, d'anciennes fosses d'extraction de graviers plus ou moins réhabilitées sont à noter à Portet-surGaronne, Sesquières, Fenouillet ou Saint-Jory.

Au final, c'est dans les secteurs périurbains en bordure immédiate du fleuve qu'ont été implantées les activités les plus génératrices de nuisances sonores (karting, gravières), olfactives (stations d'épurations), visuelles (poste électrique) et rejetées les populations « en marge de la société » (sans domiciles fixes et camps de gens du voyage). Ici, les paysages fluviaux sont soumis à de fortes pressions, et cumulent de multiples vocations : agricole, résidentielle et déversoir de l'indésirable.

\section{Conclusion}

Les paysages fluviaux urbains et périurbains toulousains reflètent différentes intentionnalités au cours de l'histoire. Les paysages fluviaux urbains du centre ancien, construits en grande partie à la fin du $\mathrm{XVIII}^{\mathrm{e}}$ siècle font partie du patrimoine et de l'identité de la ville. Ils représentent une période où les intentions étaient de protéger, valoriser et embellir les paysages fluviaux.

La tentation est grande de comparer cette période ancienne avec la période actuelle. L'échelle d’intervention n'est plus la même. Nous sommes passés de l'échelle du centre-ville ancien à l'échelle des territoires urbains et périurbains. Après la phase d'étude, la partie opérationnelle du projet «parc Garonne » vient d'être lancée au début du mois de juin 2015. Plusieurs opérations de valorisation des paysages fluviaux sont programmées jusqu'en 2020 au niveau du port de la Daurade, des quais de Tounis, de la Garonnette, du pont Saint-Michel, de l'île du Ramier et du port Viguerie. Les territoires amont et aval semblent être les oubliés de cette programmation même si un parcours découverte est programmé à Ginestous-Sesquières en aval.

L'intentionnalité de valorisation des paysages dans les espaces périurbains amont et aval affichée dans les études ne semble pas encore se diffuser largement même si à proximité immédiate du centre de

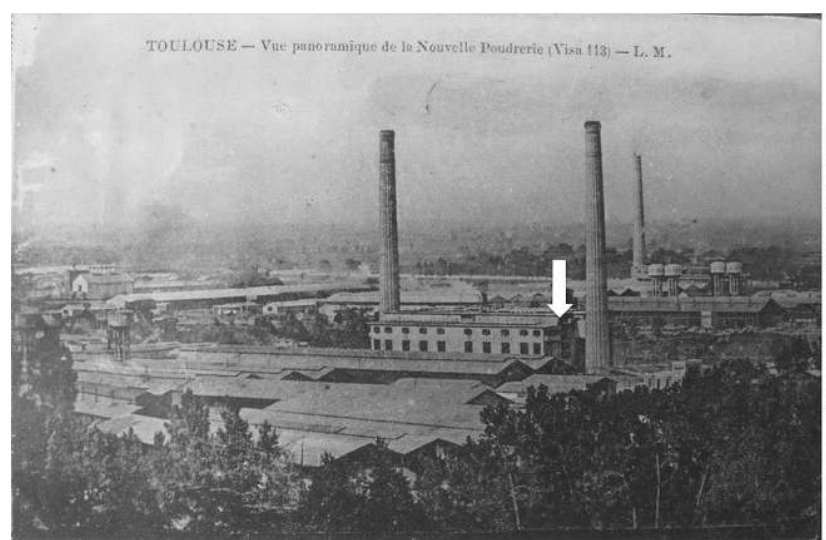

Début XXe siècle (Collection Ph Valette)

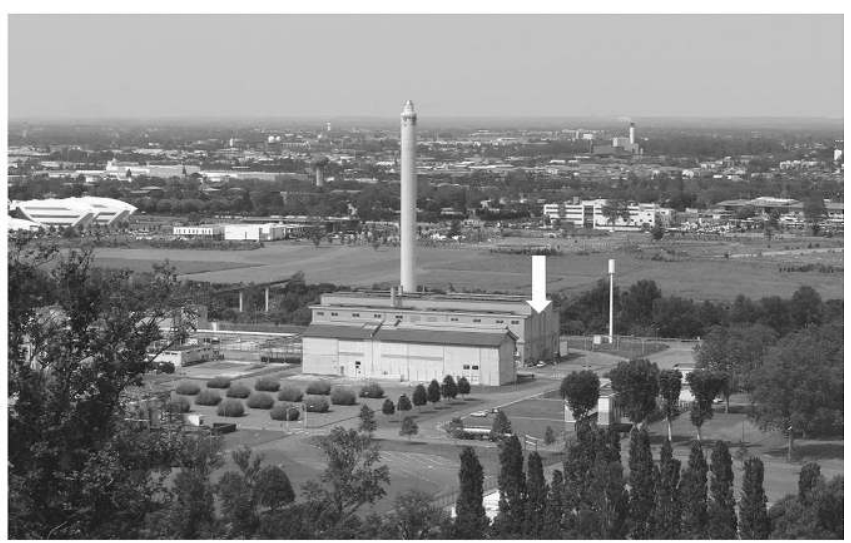

Juin 2013 (Philippe Valette)

Figure 14 : Un ancien quartier industriel en mutation : l'oncopôle de Toulouse A former industrial area in transformation: the Toulouse Oncopôle 
Toulouse des initiatives apparaissent. Dans ces territoires amont et aval, la valorisation des paysages fluviaux et le retour au fleuve semblent obéir à des opportunités déjà anciennes mises en liaison les unes aux autres.

Aujourd'hui, l'opérationnalité semble, une fois de plus, se focaliser sur le centre ancien alors que les espaces périurbains, parfois en souffrance, mériteraient un meilleur traitement paysager dans l'optique de la création du vaste "parc Garonne ». Les paysages fluviaux périurbains sont encore soumis à la pression d'une ville qui cherche à s'étendre, même en zone inondable. Néanmoins, ce caractère inondable opère une dépréciation de leur valeur, de sorte qu'ils sont aussi le réceptacle d'un certain nombre de nuisances. Or, les nuisances dans les paysages fluviaux urbains ont toujours existé (abattoirs, léproseries, hôpitaux). Ces derniers ont fait ou font l'objet de réhabilitations pour intégrer « l'arc culturel du parc Garonne ». Cependant, cette répulsion pour les espaces vulnérables au risque d'inondation est toujours d'actualité, et si elle n'a plus cours dans un centre-ville protégé par des digues, elle tend à se reporter en amont et en aval.

Jusqu'à présent, les actions situées dans le centre ancien de la ville de Toulouse faisaient de la Garonne un haut-lieu de la culture, de l'identité locale et un nouveau symbole de l'esthétique urbaine. Les parties amont et aval semblaient quelque peu à l'écart, mis à part de façon ponctuelle (espaces verts). Même si les réflexions en cours leur octroient une place plus importante dans ces territoires périurbains, en travaillant sur une échelle plus vaste, les opérations de mise en valeur semblent quelque peu les oublier, au moins jusqu'en 2020.

\section{Bibliographie}

Atelier Parisien d'Urbanisme (APUR), 2010. Paris, Métropole sur Seine, Textuel, 160 p.

Baxandall M., 1991. Formes de l'intention: sur l'explication historique des tableaux, Nîmes, Éditions Jacqueline-Chambon, coll. « Rayon Art », 272 p.

Barraud R., 2007. Approche géographique de l'intentionnalité paysagère dans les vallées de l'ouest de la France, Projets de paysage. [http://www.projetsdepaysage.frlapproche_geographique_de_l_intentionnalite_paysagere_dans_les_vallees_de_l_ouest_de_la_france_]
Beucher S., 2009. Londres 2012, événement phare ou projet de ville durable?, BAGF, Vol 86, n 3, p. 312-323.

Bonin S., 2007. Fleuves en ville : enjeux écologiques et projets urbains, Strates : matériaux pour la recherche en sciences sociales, $\mathrm{n}^{\circ} 13$, p. 185-197.

Candau J., 2003. Acteurs locaux et initiatives publiques dans le domaine du paysage, rapport du programme de recherche : " Politiques publiques et paysages », $115 \mathrm{p}$.

Carré C. (coord.), 2011. Les petites rivières urbaines d'île de France, Paris, Piren-Seine, Agence de l'eau Seine-Normandie, $85 \mathrm{p}$.

Carré C., Deutsch J.-C., 2015. L'eau dans la ville. Une amie qui nous fait la guerre, L’Aube, coll. «Essai », 319 p.

Centre Urbain d'Initiation à l'Environnement de TouLOUSE (CUIET), 1993. La vie au bord du fleuve, $72 \mathrm{p}$.

Chaline C. (dir.), 1994. Ces ports qui créèrent des villes, Paris, L'Harmattan, 299 p.

Communauté Urbaine du Grand Toulouse (CUGT), 2012. Grand parc Garonne. Plan guide à horizon 2030, 108 p.

Debarbieux B., 2007. «Actualité politique du paysage », Revue de Géographie Alpine, 95-4, p. 101-114.

Direction Générale de l'Urbanisme de l'Habitat et de la Construction (DGUHC), 2006. Le fleuve dans la ville. La valorisation des berges en milieu urbain, Les dossiers, 120 p.

Dournel S., Franchomme M., Sajaloli B., 2011. «Géohistoire d'une résurgence d'eaux troubles : les milieux humides et les cités fluviales de la France du Nord (début XIX ${ }^{\mathrm{e}}$ s., $\mathrm{Xx}^{\mathrm{e}}$ s.), Revue du Nord, $\mathrm{n}^{\circ} 26$, p. 169-187.

Dournel S., 2010. L'eau, le miroir de la ville : contribution à l'étude de la requalification urbaine des milieux fluviaux et humides (bassin parisien, Amiens, Orléans), Thèse de géographie, Université d'Orléans, 680 p.

Fortuné M., 1988. Usages passés et écologie de la Garonne, Thèse de doctorat en écologie, Université Paul-Sabatier, $162 \mathrm{p}$.

Jacob-Rousseau N., 2009. «Géohistoire/Géo-histoire : quelles méthodes pour quel récit?", Geocarrefour, Vol. 84, n 4, p. 211-216.

LAFFont J.-L., 1997. Policer la ville. Toulouse capitale provinciale au siècle des Lumières, Thèse de doctorat en histoire, Université de Toulouse Le Mirail.

Lamarre C., 2006. in Entrer en ville, actes du colloque de l'Université d'Orléans, 26-27 octobre 2001, Presses Universitaires de Rennes, 326 p.

Paisea, 2009. Cances, mars 2009, 112 p.

Papillault R., Chapel E., Pere A., 2012. Toulouse Territoires Garonne. Habiter en bord du flewve, Presses Universitaires du Mirail, 304 p.

Parent A., 2005. Entre Empire et Nation. Les représentations de la ville de Québec et de ses environs, 1760-1833, Presses Universitaires Laval, 272 p.

Romain F., 2010. « Le fleuve porteur d'images urbaines : formes et enjeux », Géocarrefour, Vol. 85 / 3, p. 253-260.

SALLES S., 2003. Le rapport à l'eau dans les projets d'aménagement urbain, Festival International de Saint-Dié.

Syndicat Mixte d’Etude et de Programmation pour L'Aménagement de la Garonne (SMEPAG), 1989. Schéma de 
protection contre les eaux de la Garonne, Tome 1, Monographie des crues de la Garonne (du pont du Roy au Bec d'Ambès), $168 \mathrm{p}$.

Topos, 2009. Water. Resource and threat, n 68, 111 p.

Valette P., Carozza J-M., Salles D., David M., Simonet G., 2014. Construction géohistorique du «sauvage » de la Garonne toulousaine : quelle part de naturalité dans les paysages fluviaux?, Développement Durable et Territoires, Vol. $5, \mathrm{n}^{\circ} 3$.

Valette P., Carozza J.-M., 2013. Toulouse face à la Garonne : emprise de l'urbanisation dans la plaine inondable et géohistoire des aménagements fluviaux, Geographicalia, 63-64, p. 207-233.

Valette P., Girou A., Carozza J.-M., Desailly B., 2013. Des balcons sur la Garonne? Évolution des paysages fluviaux périurbains aux portes nord de Toulouse, in CTHS, Comprendre les paysages urbains, $135^{\mathrm{e}}$ congrès du CTHS, Neuchâtel.
Valette P., Carozza J.-M., 2011. Les paysages fluviaux urbains : évolution du rapport ville/fleuve à travers l'exemple de l'agglomération toulousaine et la Garonne, Revue $d u$ Nord, Zones humides et villes d'hier et d'aujourd'hui : des premières cités aux fronts d'eau contemporains, Hors-série, $n^{\circ} 26$, p. 219-237.

Valette P., Carozza J.-M., 2009. Mise en ouvre d'une démarche géohistorique pour la connaissance de l'évolution des paysages fluviaux: l'exemple de la moyenne vallée de la Garonne, Geocarrefour, Vol. 84, nº 4, p. 17-27.

VALETTE P., 2002. Les paysages fluviaux de la Garonne : les métamorphoses d'un fleuve, Thèse de doctorat de géographie, UFR sciences espaces et sociétés, Université Toulouse 2, $554 \mathrm{p}$.

Valley E., 2011. Parenthèse toulousaine, quelle identité pour l'île du Ramier?, TPFE, École d'architecture et de Paysage de Bordeaux, 148 p. 